\title{
Treatment of Depression: Are Psychotropic Drugs Overdosed in Women and in the Elderly? Dosages of Psychotropic Drugs by Sex and Age in Clinical Routine
}

\section{Waldemar Greil ( $\nabla$ waldemar.greil@med.uni-muenchen.de )}

Department of Psychiatry and Psychotherapy, Ludwig Maximilian University, Munich

\section{Mateo de Bardeci}

Psychiatric Private Hospital, Sanatorium Kilchberg, Kilchberg-Zurich, Switzerland

\section{Xueqiong Bernegger}

Psychiatric Private Hospital, Sanatorium Kilchberg, Kilchberg-Zurich, Switzerland

\section{Katja Cattapan}

Psychiatric Private Hospital, Sanatorium Kilchberg, Kilchberg-Zurich, Switzerland

\section{Hans Stassen}

Institute for Response-Genetics, Psychiatric University Hospital (KPPP), Zurich

\section{Marcel Sieberer}

St. Marien-Hospital, Department of Psychiatry and Psychotherapy, University Witten/Herdecke, Hamm, Germany

\section{Renate Grohmann}

Department of Psychiatry and Psychotherapy, Ludwig Maximilian University, Munich

\section{Sermin Toto}

Department of Psychiatry, Social Psychiatry and Psychotherapy, Hannover Medical School, Hannover

\section{Research Article}

Keywords: Psychotropic drugs, Dosage, Sex, Gender, Age, Depressive Disorder, Overdose, AMSP, TDM, drug safety

Posted Date: December 9th, 2020

DOl: https://doi.org/10.21203/rs.3.rs-116497/v1

License: (c) (i) This work is licensed under a Creative Commons Attribution 4.0 International License. Read Full License 


\section{Abstract}

Background:

Psychotropic drugs show higher concentration-dose ratios in women and in the elderly. Furthermore, women partially exhibit more adverse drug reactions than men. Lower dosages in most psychotropic drugs may be recommended in women and in the elderly. This study describes prescribed dosages of psychotropic drugs in depressive patients across sexes and age groups in clinical routine.

Method:

In 32'082 inpatients with depressive disorders (data acquired by the European drug safety program AMSP), the influence of sex and age on prescribed dosages are analysed for the 10 most commonly prescribed drugs in our dataset (descending in the frequency of prescription: mirtazapine, venlafaxine, lorazepam, quetiapine, citalopram, escitalopram, olanzapine, duloxetine, zopiclone and sertraline) and additionally zolpidem. Confounding variables for sex differences, such as severity of depression and age distribution are examined. Dose decline in patients over 65 years is calculated. The observed sex and age differences in prescriptions are compared to differences in pharmacokinetic literature.

Results

Among patients over 65 , a statistically significant decrease in dosages for increasing age (between $0.65 \%$ and $2.83 \%$ every year of age) was observed, except for zopiclone. On the other hand, only slight or no influence of sex in prescribed dosages was found.

Conclusion

Age definitely influences physicians' decisions in most drugs, but to a lower extent than pharmacokinetic data suggests. Although lower dosages of psychotropic drugs are appropriate for females according to pharmacokinetic data, they are usually prescribed the same dosage as males. Thus, various psychotropic drugs may be overdosed in women.

\section{Background}

At the same dosage, women show higher plasma levels than men for numerous drugs [1-3]. This is due to sex differences in height, body weight, fat proportion, kidney function, the activity of CYP 450 enzymes and the frequency of smoking [4-9]. Sex differences in pharmacokinetics with a significantly higher concentration-dose ratio in women have been proven for a number of psychotropic substances, e.g. mirtazapine [1, 10], olanzapine $[2,11]$, venlafaxine $[1,12-14]$, escitalopram $[1,15]$ and duloxetine [16].

Furthermore, despite ambiguous results $[17,18]$ women report a higher rate of adverse drug reactions (ADRs) $[3,19,20]$. 
Although elderly generally suffer from ADRs more often [21], in the case of psychotropic drugs a number of studies under naturalistic conditions showed similar percentages of severe ADRs in elderly as in young patients $[20,22,23]$. On the other hand, an increase of concentration-dose ratios with age is described for various psychotropic drugs, e.g. mirtazapine [1, 10], venlafaxine [1, 12-14], quetiapine [4, 11, 24], citalopram [1, 10, 25], escitalopram [1], olanzapine [2, 11], zopiclone [26] and sertraline [1, 10]. Since agerelated changes in body composition and polypharmacy lead to different pharmacokinetics and pharmacodynamics in the elderly, the recommendation to lower the dosage of psychotropic drugs in the elderly is generally accepted [27-29, 29a].

Although women are the main users of psychotropic drugs [30], they are underrepresented in treatment studies, and therefore treatment guidelines are mainly based on findings in male patients [31]. According to Sørup et al. [32], the influence of patient's sex on ADRs is an understudied factor.

In 2013, the U.S. Food and Drug Administration (FDA) approved label changes specifying new maximal dosing recommendations for zolpidem, being $5 \mathrm{mg} /$ day for females and $10 \mathrm{mg} /$ day for males [33, 34]. According to the FDA, higher dosages could lead to next-morning impairments, especially driving impairment [33,34]. However, this guidance is controversial. It has been argued that a disturbed sleep by underdosed zolpidem in females may impair driving even more than the intake of the $10 \mathrm{mg} / \mathrm{day}$ dose [18]. This controversy motivated our inclusion of zolpidem in our study.

In this study, a large data set from a pharmacovigilance project is used to test whether in clinical routine the dosage of psychotropic drugs in the treatment of depression is lower in females than in males. We selected the 10 most prescribed drugs in our dataset and additionally zolpidem. Furthermore, we also analyse to which extent the dosages of psychotropic drugs are influenced by age in general and within the group of elderly.

\section{Method}

\section{Data source}

The analysed prescription data of the present study was gathered in the project "Arzneimittelsicherheit in der Psychiatrie" (AMSP; drug safety program in psychiatry). AMSP is an ongoing European multi-center drug safety program which collects data on psychopharmacotherapy and severe adverse drug reactions from psychiatric hospitals in a naturalistic setting since 1993. AMSP's pharmacovigilance methods have been described in detail previously [35-37]. Briefly, AMSP consists of two principal data collections (prescription data and severe adverse drug reactions) from 116 hospitals so far in Germany, Switzerland and Austria, as well as temporarily from one hospital each in Belgium and Hungary. The number of participating hospitals increased from nine in 1994 to 63 in 2015. In a cross-sectional approach, all participating hospitals record drug prescriptions for all inpatients under surveillance on two reference days per year. All drugs administered on these days are assessed along with the patients' age, sex and leading psychiatric diagnosis. Evaluations of the AMSP database have been approved by the Ethics 
Committee of the University of Munich and the Ethics Committee of the Hannover Medical School (Nr. 8100 BO S 2018). This study adheres to the Declaration of Helsinki and its later amendments. The AMSP program is a continuous observational post marketing drug surveillance program and does not interfere with the ongoing clinical treatment of patients under surveillance.

\section{Sample description}

32'082 patients older than 17 and younger than 90 years of age, hospitalized between 2001 and 2015, with a leading admission diagnosis of major depressive disorder (MDD) are investigated. The dosages of the 10 most prescribed drugs and zolpidem are analysed (descending in the frequency of prescription): mirtazapine $\left(n=10^{\prime} 431\right)$, venlafaxine $\left(n=8^{\prime} 072\right)$, lorazepam $\left(n=7^{\prime} 757\right)$, quetiapine $\left(n=6^{\prime} 993\right)$, citalopram ( $\left.n=3^{\prime} 909\right)$, escitalopram ( $\left.n=3^{\prime} 842\right)$, olanzapine $\left(n=3^{\prime} 441\right)$, duloxetine $\left(n=3^{\prime} 070\right)$, zopiclone $\left(n=2^{\prime} 936\right)$, sertraline $\left(n=2^{\prime} 909\right)$ and zolpidem $\left(n=1^{\prime} 873\right)$. The sample consists of $11^{\prime} 887(37.1 \%)$ males and 20'195 $(62.9 \%)$ females. Note that a patient may have had more than one psychotropic drug prescribed. Table 1 shows a summary of the sample composition.

Table 1

Sample Composition

\begin{tabular}{|lc|}
\hline Sample Composition & \\
\hline Total number of patients & $32^{\prime} 082$ \\
\hline Number of Males & $11^{\prime} 887(37.1 \%)$ \\
\hline Number of Females & $20^{\prime} 195(62.9 \%)$ \\
\hline Age males in years (mean \pm S.D.) & $49.5 \pm 15.3$ \\
\hline Age females in years (mean \pm S.D.) & $51.9 \pm 16.5$ \\
\hline Number of psychotropic prescriptions per patient (mean \pm S.D.) & $2.6 \pm 1.3$ \\
\hline S.D.: standard deviation & \\
\hline
\end{tabular}

When analysing prescribed dosage differences between sexes, it is important to identify possible confounding variables that could account for distortions in the analysis. In this study, we examined the following possible confounding variables: severity of MDD, differences along calendar years and differences in age distribution.

In figure 1, the number of male and female patients along calendar years are depicted. We do not see any substantial difference in the ratio between male and female patients over time.

Table 2 shows no substantial differences in the severity of cases for male and female patients. Table 3 gives the severity of cases for young and elderly patients. In the small group of psychotic MDD (13.4\%), 
there is a higher percentage in the elderly than in younger patients (18.0\% versus $12.2 \%)$. Nevertheless, we assume that severity and differences over years cannot distort our analysis to a significant extent. On the other hand, the age distributions (depicted in figure 2) differ substantially. In particular, there are proportionally more elderly women than elderly men. Therefore, we need to adjust our analysis in order to take into account differences in age distributions.

Table 2

Severity of cases by sex

\begin{tabular}{|c|c|}
\hline \multicolumn{2}{|l|}{ Severity of cases by sex } \\
\hline Mild MDD ("F32.0","F33.0") & $368(1.2 \%)$ \\
\hline \multirow{2}{*}{ Male } & $151(1.3 \%)$ \\
\hline & $217(1.1 \%)$ \\
\hline Moderate MDD ("F32.1","F33.1" & $8^{\prime} 237(25.7 \%)$ \\
\hline Male & $3^{\prime} 033(25.5 \%)$ \\
\hline Female & $5^{\prime} 204(25.8 \%)$ \\
\hline Severe MDD ("F32.2", "F33.2") & $18^{\prime} 257(56.9 \%)$ \\
\hline Male & $6720(56.5 \%)$ \\
\hline Female & $11^{\prime} 537(57.1 \%)$ \\
\hline Psychotic MDD ("F32.3", "F33.3") & 4’308 (13.4\%) \\
\hline Male & $1 ` 645(13.8 \%)$ \\
\hline Female & $2^{\prime} 663(13.2 \%)$ \\
\hline No info or other MDD ("F32.8","F32.9","F33.4","F33.8,"F33.9") & $912(2.8 \%)$ \\
\hline Male & $338(2.8 \%)$ \\
\hline Female & $574(2.8 \%)$ \\
\hline $\begin{array}{l}\text { Diagnoses according to ICD-10 ( } 10 \text { th revision of the Internatio } \\
\text { Diseases and Related Health Problems). The severity of cases } \\
\text { female patients. }\end{array}$ & $\begin{array}{l}\text { ification of } \\
\text { leen male and }\end{array}$ \\
\hline
\end{tabular}




\begin{tabular}{|c|c|}
\hline Mild MDD ("F32.0","F33.0") & $368(1.1 \%)$ \\
\hline Up to 65 ys & $287(1.3 \%)$ \\
\hline Over 65 ys & $81(1.2 \%)$ \\
\hline Moderate MDD ("F32.1","F33.1") & $8^{\prime 2} 237$ (25.7\%) \\
\hline Up to 65 ys & 6‘803 (26.8\%) \\
\hline Over 65 ys & $1 ` 434(21.4 \%)$ \\
\hline Severe MDD ("F32.2", "F33.2") & $18^{\prime} 257(56.9 \%)$ \\
\hline Up to 65 ys & $144^{\prime} 501(57.1 \%)$ \\
\hline Over 65 ys & $3 ’ 756(56.1 \%)$ \\
\hline Psychotic MDD ("F32.3", "F33.3") & 4’308 (13.4\%) \\
\hline Up to 65 ys & 3’104 (12.2\%) \\
\hline Over 65 ys & 1'204 (18.0\%) \\
\hline No info or other MDD ("F33.4","F32.9","F32.8","F33.9","F33.8") & $912(2.8 \%)$ \\
\hline Up to 65 ys & $693(2.7 \%)$ \\
\hline Over 65 ys & 219 (3.3\%) \\
\hline
\end{tabular}

\section{Statistical analysis}

The statistical analysis was performed using $\mathrm{R}$ version 3.6.2. To assess the influence of age in the decision of prescribed dosages, the slope of a linear fit among patients older than 65 and younger than 90 years of age is computed. To estimate its significance, a t-test is performed on the coefficient of the slope under the hypothesis to be zero. Along with the slope, also the percentage change in relation to the previous year is computed, which is calculated from the coefficient of an exponential fit. Since in pharmacokinetic literature the age differences are often compared between age groups of up to 65 years old and over 65 years old, we also compared the mean dosage differences between these age groups in a table, with the elderly group chosen as reference. We compute the p-value from a Wilcoxon test. 
In order to account for the sex differences in dosages, we adjust for age by comparing prescriptions in patients with similar age. We divide the patients by sex and assign them to 24 age groups of three consecutive years, and we calculate the mean dosage in each group for both genders separately. Then, we calculate the sex difference within each age group, where female patients are chosen as reference. Finally, the total sex difference is calculated as the weighted mean of all percentage differences, by weighting with the number of patients (male and female) of each age group. We depicted the analysis by plotting the daily prescribed doses in relation to patients' age in bars of six consecutive years. To calculate the significance, we run a paired Wilcoxon signed-rank test, in which the input are the mean values of male and female of the 24 age groups.

\section{Results}

\section{Age}

The results of the influence of age in dosage prescription are depicted in figure 3 with antidepressants, figure 4 with antipsychotics and figure 5 with hypnotics and tranquilizers. Several bar plots can be seen, in which the height of the green bars gives the mean daily prescribed doses in mg including both sexes, and the $x$-axis gives the age of the patients. Further, a red line is depicting a linear fit in patients over 65 years old. The slope of the fit is computed in the upper left corner, along with the percentage change. The percentage change is the average percentage increase (or decrease) of daily dosage in relation to the previous year of age. Along with the slope and the percentage change, the $p$-value under the hypothesis of zero slope is computed. Further, in table 4 the mean differences of dosages between patients up to 65 years and older than 65 years is shown. This description holds for each of the following three sections. 
Table 4

Mean dosage differences between patients up to 65 and older than 65 years

\begin{tabular}{|c|c|c|c|c|c|c|c|c|}
\hline \multirow[t]{3}{*}{ Drug } & \multicolumn{3}{|c|}{ Over $17 y s$ to $65 y s$} & \multicolumn{3}{|c|}{ Over 65ys to $89 y s$} & \multirow{3}{*}{$\begin{array}{l}\text { Difference } \\
\text { [\%](means) }\end{array}$} & \multirow{3}{*}{$\begin{array}{l}\text { p-value } \\
\text { (means) }\end{array}$} \\
\hline & $\mathbf{N}$ & Mean dose & SE & $\mathbf{N}$ & Mean dose & SE & & \\
\hline & & \multicolumn{3}{|l|}{ [mg/d] } & \multicolumn{2}{|l|}{ [mg/d] } & & \\
\hline Citalopram & 3130 & 29.2 & 0.2 & 779 & 27.3 & 0.5 & +7.0 & $<0.001$ \\
\hline Escitalopram & 3143 & 16.3 & 0.1 & 699 & 14.4 & 0.3 & +13.4 & $<0.001$ \\
\hline Sertraline & 2357 & 102.8 & 1.0 & 552 & 93.6 & 2.0 & +9.7 & $<0.001$ \\
\hline Mirtazapine & 7419 & 33.2 & 0.2 & 3012 & 33.6 & 0.3 & NS & NS \\
\hline Venlafaxine & 6587 & 200.5 & 1.1 & 1485 & 175.9 & 2.2 & +14.0 & $<0.001$ \\
\hline Duloxetine & 2462 & 79.9 & 0.6 & 608 & 73.0 & 1.2 & +9.4 & $<0.001$ \\
\hline Olanzapine & 2601 & 10.2 & 0.1 & 840 & 8.2 & 0.2 & +24.4 & $<0.001$ \\
\hline Quetiapine & 5673 & 215.1 & 2.6 & 1320 & 177.8 & 4.4 & +21.0 & $<0.001$ \\
\hline Zolpidem & 1379 & 10.5 & 0.1 & 494 & 9.5 & 0.1 & +10.4 & $<0.001$ \\
\hline Zopiclone & 2162 & 7.2 & 0.1 & 774 & 7.2 & 0.1 & NS & NS \\
\hline Lorazepam & 5624 & 1.9 & 0.0 & 2133 & 1.5 & 0.0 & +29.7 & $<0.001$ \\
\hline \multicolumn{9}{|c|}{$\begin{array}{l}\text { Number of patients }(\mathrm{N}) \text {, prescribed mean dosages and standard error }(\mathrm{SE}) \text { for antidepressant, } \\
\text { antipsychotic and hypnotic drugs in inpatients up to } 65 \text { and over } 65 \text { years old. On the right hand the } \\
\text { difference between means as percentage change is shown, in which the elderly patient group is } \\
\text { chosen as reference. The p-value from a Wilcoxon test is calculated. ys: years of age }\end{array}$} \\
\hline
\end{tabular}

\section{Age: Antidepressants}

In the six analysed antidepressants (Fig. 3), we found for each additional year of age a slight increase of daily dose in young patients, a plateau in middle aged patients and a decrease in elderly patients. Among patients older than 65 years, a decrease between $0.65 \%$ for the noradrenergic and specific serotonergic antidepressant drug (NaSSA) mirtazapine and $1.32 \%$ for the selective serotonin reuptake inhibitor (SSRI) sertraline for every increasing year can be seen. For the SSRI citalopram and escitalopram, we found a decrease of $0.68 \%$ and $1.13 \%$, respectively, and for the serotonin and norepinephrine reuptake inhibitors (SNRI) venlafaxine and duloxetine a decrease of $1 \%$ and $0.76 \%$, respectively. The results of all these antidepressants are statistically significant at a level of 0.01 . Comparing the mean prescribed dosages between patients up to 65 years old and older than 65 years old, we found statistically significant differences except for mirtazapine, varying from $7 \%$ for citalopram to $14 \%$ for venlafaxine (Table 4 ).

\section{Age: Antipsychotics}


In figure 4 for both antipsychotics, olanzapine and quetiapine, a plateau can be seen in young and middle age patients. However, a more pronounced slope than in antidepressants can be seen in elderly patients. Within patients over 65 years old, we found a decrease of $2.48 \%$ and $2.83 \%$ in daily prescribed dosages for each increasing year for olanzapine and quetiapine respectively $(p<0.0001)$. Comparing the mean prescribed dosages between patients up to 65 years old and older than 65 years old, we found statistically significant differences of $24.4 \%$ for olanzapine and $21 \%$ for quetiapine (Table 4 ).

\section{Age: Hypnotics and Tranquilizers}

Between the two hypnotic drugs (zopiclone and zolpidem) and the tranquilizer drug lorazepam depicted in figure 5 , the distribution of the daily prescribed dosages differs substantially. In the case of zolpidem (Figure 5a), a slight increase can be seen in young patients, a plateau in middle aged patients and a decrease in elderly patients. This distribution is similar to the distribution of antidepressants. For zopiclone (Figure $5 \mathrm{~b}$ ), a constant dosage around $7.2 \mathrm{mg}$ per day can be seen for all ages. Therefore, age does not seem to influence prescribed dosages in the case of zopiclone. On the other hand, for lorazepam (Figure 5c), a steady reduction of prescribed dosages can be seen across all ages.

Within patients over 65 years old, we found a decrease of $1.17 \%$ and $1.26 \%$ in daily prescribed doses for each increasing year of age for zolpidem and lorazepam respectively $(p<0.001)$. In the case of zopiclone, we did not find statistically significant differences with increasing age. When comparing the mean difference in prescribed dosages between patients up to 65 years old and older than 65 years old, statistically significant differences were found for zolpidem (10.4\%) and lorazepam (29.7\%), but not for zopiclone (Table 4).

\section{Sex}

The results for sex influence in prescribed dosages is given in figure 6 with antidepressants, figure 7 with antipsychotics and figure 8 with hypnotics and tranquilizers. Several plots can be seen, where the y-axis gives the prescribed daily dose and the $x$-axis the age. Doses of males are depicted in blue and of females in orange, the standard errors are depicted in red. On the upper left corner further statistics can be found: the $p$-value under the hypothesis, that there is no difference between sexes; furthermore, the difference between sexes computed as percentage change, where females are chosen as reference. This description holds for each of the following three sections.

\section{Sex: Antidepressants}

In the case of antidepressants (figure 6), we found statistically significant differences in daily dose prescription between sexes for escitalopram and venlafaxine, in which males received respectively $5.13 \%$ 
and $4.59 \%$ more than females. For citalopram, sertraline, mirtazapine and duloxetine we did not find statistically significant differences between sexes.

\section{Sex: Antipsychotics}

Regarding antipsychotics (Figure 7), we found statistically significant differences for olanzapine, in which males receive $4.94 \%$ more than females. For quetiapine, we did not find statistically significant differences.

\section{Sex: Hypnotics and Tranquilizers}

Among the hypnotic drugs zolpidem and zopiclone, and the tranquilizer lorazepam (Figure 8), we did not find statistically significant differences between males and females.

\section{Discussion}

\section{Age}

In order to discuss our findings, we present a literature review about age and sex influence on plasma levels of patients taking psychotropic drugs and compare it to the influence of age and sex on prescribed dosages in our findings. A comprehensive table describing bullet points of the different studies in literature can be found in the supplementary material (Additional file 1; Table 1 and 2 supplementary). In the following sections only the key studies in literature are cited and compared to our findings.

\section{Age: Antidepressants}

Reis et al. [1] analysed in 2009 the plasma levels for citalopram and escitalopram at steady state of 2330 and 1470 subjects respectively, finding that elderly patients over 64 years old had $84 \%$ and $91 \%$ higher plasma levels than younger patients at the same dosage. Whereas, in the prescribed dosages we only found a difference of $7.0 \%$ and $13.4 \%$. For sertraline, Reis et al. [1] found a difference of $31 \%$ in 1071 patients, and $44 \%$ for mirtazapine in 660 patients. In contrast, for sertraline we only observed a difference of $9.7 \%$ in prescribed dosages, and for mirtazapine we did not find statistically significant results. Reis et al. [1] also analysed the plasma levels of venlafaxine. They found at the same dosage $38 \%$ difference in plasma levels between elderly and young patients whereas we only found a difference of $14 \%$.

For duloxetine, we found a significant decrease in dosage prescription within the elderly and significant differences between young and elderly. In comparison, a study of Lobo et al. [16] computed a fit over all ages and found a slope of -0.33 for apparent total clearance. Using the same method for the description of dosages in our data, the analogue slope shows -0.146 (Additional file 2, Fig. 1 supplementary). The slopes need to be normalized in order to be compared, which yields -0.50 for the apparent clearance, and 
-0.17 for dosage prescription. Again, the influence of age on prescription of duloxetine is substantially smaller than the influence on the plasma level according to the literature.

The case of mirtazapine is particularly noteworthy, since we found no differences in the prescription between young and elderly patients, and literature shows an increase of plasma levels in elderly above $40 \%$. Although we found statistically significant differences between prescribed dosages in young and elderly patients for the remaining antidepressants, the differences in pharmacokinetic literature are clearly more pronounced than in real clinical decisions.

\section{Age: Antipsychotics}

Regarding the antipsychotics olanzapine and quetiapine, we found statistically significant mean differences in prescribed dosages of $24.4 \%$ and $21 \%$ respectively (between patients up to 65 and over 65 years old). In literature, the differences in pharmacokinetics are more pronounced: Castberg et al. [11] found $75 \%$ higher olanzapine plasma levels in elderly analysing 6239 patients and $35 \%$ for quetiapine in 1949 patients. Bakken et al. [24] found 50\% higher levels analysing 601 patients for quetiapine (see also Additonal File 2, Fig.1 and 2, suppl.).

\section{Age: Hypnotics and Tranquilizers}

For the analysed hypnotics and tranquilizers, we did not find literature describing pharmacokinetic data at steady state, but for single dose. For zolpidem, dosage prescriptions in our dataset are only $10.4 \%$ higher in young patients, whereas Olubodun et al. [38] found more pronounced age differences: in males $133 \%$ in the maximum concentration $\left(C_{\max }\right), 80 \%$ in half-life and $264 \%$ in the Area Under the Curve (AUC); in females $80 \%$ in $\mathrm{C}_{\max }$ and $60 \%$ in AUC. No significant differences have been found by Olubodun et al. [38] in half-life between younger and elderly females [38].

In the case of zopiclone, Gaillot et al. [26] found increasing differences with increasing age. Elderly between 60 and 68 years old had 31\% higher AUC, and elderly between 74 and 85 years old had $105 \%$ higher AUC. We did not find any influence of age in dosage prescriptions of zopiclone. In particular, we found a flat distribution with daily doses around $7.2 \mathrm{mg} /$ day.

For lorazepam, Greenblatt et al. [40] found no significant influence of age in elimination half-life $(n=30)$, but a total clearance of $22 \%$ less in elderly patients. We found a difference in dosage prescriptions of $30 \%$ between young and elderly patients.

In summary, for zolpidem and zopiclone physicians take into account the kinetic data only to a limited extent. In the case of lorazepam, there is not enough pharmacokinetic data to draw conclusions.

\section{Sex}


A comprehensive table describing bullet points of the different studies in literature about sex differences in plasma levels of psychotropic drugs is given in the supplementary material (Additional file 1; Table 2 supplementary).

\section{Sex: Antidepressants}

Although pharmacokinetic data of several antidepressants is surprisingly limited, we found overall much higher differences in plasma levels between sexes in pharmacokinetic literature than in real clinical decisions. In 2009, Reis et al. [1] analysed the plasma levels for citalopram and escitalopram at steady state of 2330 and 1470 subjects respectively, and reported that female patients have $25 \%$ and $9 \%$ higher plasma levels than men at the same dosage. In the prescribed dosages we only found a difference of $1.6 \%$ and $5.1 \%$. Also Scherf-Clavel et al. [15] examined 114 patients with prescriptions of escitalopram finding a sex difference in dose-corrected serum concentration of $39.6 \%$ in patients up to 65 years and of $45.9 \%$ in patients over 65 years old.

Reis et al. [1] also investigated the plasma levels of 660 patients with mirtazapine prescriptions and 716 with venlafaxine prescriptions. They found plasma level differences of $16 \%$ and $42 \%$, whereas we only found a difference of $2.8 \%$ and $4.6 \%$ in prescribed dosages. Sigurdsson et al. [13] also studied 1417 patient with venlafaxine prescriptions and found at steady state that women have $18.8 \%$ higher doseadjusted serum concentrations.

For sertraline, we found a dosage difference of $1.4 \%$. This case is consistent with the results of Reis et al. [1] in which they did not find statistically significant differences in plasma levels for sertraline in 1071 patients. For duloxetine, Lobo et al. [16] estimated the pharmacokinetic sex difference to be $64 \%$ for the same dosage, which is in part explained by differences in smoking habits. In contrast, we only found a $2.8 \%$ dose difference in real clinical decisions.

Overall, the difference in dosage prescriptions between sexes for the six most prescribed antidepressants does not reflect pharmacokinetic data except for sertraline, since there was no difference found in prescribed dosages as well as in plasma levels.

\section{Sex: Antipsychotics}

For olanzapine, Castberg et al. [11] found a $26.1 \%$ higher dose adjusted plasma concentration in women than in men for 5826 patients on stable dose. In contrast, we found a prescribed dosage difference of only $4.9 \%$.

For quetiapine, Bakken et al. [24] investigated 601 patients and did not find evidence that sex influences pharmacokinetics. Similarly, we did not find evidence that sex influences prescribed dosages. On the other hand, Castberg et al. [11] examined 2210 patients with quetiapine prescriptions and did not find evidence of sex differences in plasma levels for young patients, but with increasing age, the 
pharmacokinetic differences become noticeable and significant. In our study, we did not find evidence of increasing dosages differences with increasing age in real clinical conditions.

Similarly to antidepressants, for antipsychotics we found literature with evidence of much higher pharmacokinetic sex differences than naturalistic differences in dosage prescriptions.

\section{Sex: Hypnotics and Tranquilizers}

For the benzodiazepine lorazepam, we surprisingly found very limited literature about its pharmacokinetics. There is a study of Greenblatt et al. from 1979 [40], where 30 patients are analysed with a single intravenous dose. No apparent relation between sex and pharmacokinetics was found. Similarly, we did not find evidence of sex affecting dosage prescriptions of lorazepam.

Greenblatt et al. [40] also conducted a study in 2019 in which the clearance of zolpidem in 70 patients was analysed. Although significant differences between sexes in terms of pharmacokinetics was found, according to the study, no evidence of sex related effectiveness nor adverse reaction was found. In our study, we did not find evidence for sex related prescription differences. For the dosage prescription of zolpidem, it is not clear whether sex should be considered. Nevertheless, the results of our study show that physicians in Germany, Switzerland and Austria tend to prescribe zolpidem without considering the FDA recommendations of a maximum dose of $5 \mathrm{mg}$ for women (mean dose for both sexes about $10 \mathrm{mg}$ per day).

Regarding zopiclone, Gaillot et al. 1983 [39] found that $C_{\max }$ is $14 \%$ to $24 \%$ higher, and AUC is $16 \%$ to $20 \%$ higher in women than men. In contrast, we found no evidence of sex influencing the decision of dosage prescription.

Overall, we found very old and limited pharmacokinetic literature about the two most prescribed hypnotics/tranquilizers and zolpidem. There is certainly room for research in this field.

\section{Limitations}

Several limitations of the study must be noted. First, the diagnosis of depression was established in clinical routine instead of standardised diagnostic procedures. Secondly, no data are available of the clinical condition, possible comorbidity and duration of treatment. Thirdly, the plasma levels of the patients are not present, instead mean values are taken from the literature. Furthermore, the study is based on the assumption that plasma levels are valid parameters for dosage decision. Finally, only hospitalized patients suffering from depression were examined. The strength of the naturalistic study is the large database reflecting clinical reality.

\section{Conclusion}


In conclusion, we found that patients' age does influence physician decisions, but to a lower extent than what pharmacological literature would suggest. On the other hand, we found that sex overall does not influence physician decision-making. Further, the plasma level differences found in pharmacokinetic literature between sexes are overall much larger than the prescribed dosages differences. We recall, patients body weight is not present in the dataset we analysed. Therefore, the results are not adjusted for weight. According to the Federal Statistical Office of Germany, males have an average body weight of $85 \mathrm{~kg}$ and women of $68.7 \mathrm{~kg}$ [41]. Men have in average $23.7 \%$ more body mass than women [41]. Hence, not only the sex differences in pharmacokinetics are not taken into account, but also sex differences in body mass do not seem to influence clinical decisions. Overall, the results of our study imply two possibilities: either plasma levels are not as correlated to drug effectiveness and adverse effects as is generally believed, or there is an underestimation of biological sex differences on behalf of physicians. The latter interpretation may also explain the partially disproportional number of adverse reactions in women. Although there is a large overlap in biological parameters between sexes, mean plasma levels differ substantially. However, we do not see these sex differences in the mean dosages. There is an urgent need for studies to examine whether adjusting the dosage in women on the basis of blood levels leads to better efficacy and lower frequency of adverse effects than the "treatment as usual" with similar dosages for both sexes.

\section{Declarations}

\section{Ethics approval and consent to participate}

Evaluations of the AMSP database have been approved by the Ethics Committee of the University of Munich and the Ethics Committee of the Hannover Medical School (Nr. 8100 BO S 2018). This study adheres to the Declaration of Helsinki and its later amendments. The AMSP program is a continuous observational post marketing drug surveillance program and does not interfere with the ongoing clinical treatment of patients under surveillance. Hence, all methods (statistical analyses) were carried out in accordance with relevant guidelines and regulations.

(All experimental protocols were approved by the named institutions: Not applicable, since no experiments).

(Confirming that informed consent was obtained from all subjects: Not applicable, since only anonymised data was used,)

\section{Consent for publication:}

Not applicable (all authors agreed with the publication).

\section{Availability of data and materials:}




\section{Competing interests}

ST is a member of the advisory board for Otsuka and Janssen-Cilag and has received speaker's honoraria from Janssen-Cilag, Lundbeck/Otsuka and Servier. All other authors state they have no conflicts of interest to declare.

\section{Role of the funding source}

The authors did not receive any specific grants or funding for the present study. The AMSP drug safety project is facilitated by non-profit associations in Germany, Austria, and Switzerland. The AMSP project has been supported with unrestricted educational and research grants since 1993 by the following companies:

German companies: Abbott GmbH \& Co. KG, AstraZeneca GmbH, Aventis Pharma Deutschland GmbH GE-O/R/N, Bayer Vital GmbH, Boehringer Mannheim GmbH, Bristol-Myers-Squibb, Ciba Geigy GmbH, Desitin Arzneimittel GmbH, Duphar Pharma GmbH \& Co. KG, Eisai GmbH, Esparma GmbH Arzneimittel, GlaxoSmithKline Pharma GmbH \& Co. KG, Hoffmann-La Roche AG Medical Affairs, Janssen-Cilag GmbH, Janssen Research Foundation, Knoll Deutschland GmbH, Lilly Deutschland GmbH Niederlassung Bad Homburg, Lundbeck GmbH \& Co. KG, Novartis Pharma GmbH, Nordmark Arzneimittel GmbH, Organon $\mathrm{GmbH}$, Otsuka-Pharma Frankfurt, Pfizer GmbH, Pharmacia \& Upjohn GmbH, Promonta Lundbeck Arzneimittel, Recordati Pharma GmbH, Rhone-Poulenc Rohrer, Sanofi-Synthelabo GmbH, Sanofi-Aventis Deutschland, Schering AG, SmithKlineBeecham Pharma GmbH, Solvay Arzneimittel GmbH, Synthelabo Arzneimittel GmbH, Dr. Wilmar Schwabe GmbH \& Co., Thiemann Arzneimittel GmbH, Troponwerke GmbH \& Co. KG, Upjohn GmbH, Wander Pharma GmbH, and Wyeth-Pharma GmbH.

Austrian companies: Astra Zeneca Österreich $\mathrm{GmbH}$, Boehringer Ingelheim Austria, Bristol-Myers Squibb $\mathrm{GmbH}, \mathrm{CSC}$ Pharmaceuticals $\mathrm{GmbH}$, Eli Lilly $\mathrm{GmbH}$, Germania Pharma GmbH, GlaxoSmithKline Pharma $\mathrm{GmbH}$, Janssen-Cilag Pharma GmbH, Lundbeck $\mathrm{GmbH}$, Novartis Pharma $\mathrm{GmbH}$, Pfizer Med Inform, and Wyeth Lederle Pharma GmbH.

Swiss companies: AHP (Schweiz) AG, AstraZeneca AG, Bristol-Myers Squibb AG, Desitin Pharma GmbH, Eli Lilly (Suisse) S.A., Essex Chemie AG, GlaxoSmithKline AG, Janssen-Cilag AG, Lundbeck (Suisse) AG, Organon AG, Pfizer AG, Pharmacia, Sanofi-Aventis (Suisse) S.A., Sanofi-Synthelabo SA, Servier SA, SmithKlineBeecham AG, Solvay Pharma AG, Wyeth AHP (Suisse) AG, and Wyeth Pharmaceuticals AG.

\section{Authors' contributions}


W.G. and S.T. initiated the study. W.G. and M.de B. wrote the main manuscript text. M.de B. conducted the final statistical analyses and prepared figures and tables. X.B. carried out the preliminary statistical analyses. H.S. designed the database for the evaluation and supervised the statistics. A.W. undertook the literature research. A.W., K.C., M.S., H.S., R.G and S.T. gave suggestions for improvement of the various versions of the manuscript. All authors reviewed and accepted the final version.

\section{Acknowledgements}

The authors would like to thank all psychiatric hospitals participating in the AMSP project, in particular the drug monitors, for their continuous support in data collection. Thanks also to Mrs. Jamila Willms, Bachelor of Science (Psychology), for her careful checking and editing of the manuscript including additional literature Research.

\section{References}

1. Reis $\mathrm{M}$, Aamo T, Spigset $\mathrm{O}$, Ahlner J. Serum concentrations of antidepressant drugs in a naturalistic setting: compilation based on a large therapeutic drug monitoring database. Ther Drug Monit. 2009;31(1):42-56. https://doi.org/10.1097/FTD.0b013e31819114ea.

2. Weiss U, Marksteiner J, Kemmler G, Saria A, Aichhorn W. Effects of age and sex on olanzapine plasma concentrations. J Clin Psychopharmacol. 2005;25(6):570-4. https://doi.org/10.1097/01.jcp.0000185427.08268.db.

3. Zucker I, Prendergast BJ. Sex differences in pharmacokinetics predict adverse drug reactions in women. Biol Sex Differ. 2020;11:32. https://doi.org/10.1186/s13293-020-00308-5.

4. Aichhorn W, Marksteiner J, Walch T, Zernig G, Saria A, Kemmler G. Influence of age, gender, body weight and valproate comedication on quetiapine plasma concentrations. Int Clin Psychopharmacol. 2006a;21(2):81-5. https://doi.org/10.1097/01.yic.0000188213.46667.f1.

5. Aichhorn W, Whitworth AB, Weiss EM, Marksteiner J. Second-generation antipsychotics: Is there evidence for sex differences in pharmacokinetic and adverse effect profiles? Drug Saf. 2006b;29(7):587-98. https://doi.org/10.2165/00002018-200629070-00004.

6. Meibohm B, Beierle I, Derendorf H. How Important Are Gender Differences in Pharmacokinetics? Clin Pharmacokin. 2002;41(5):329-42. https://doi.org/10.2165/00003088-200241050-00002.

7. Oliveira P, Ribeiro J, Donato H, Madeira N. Smoking and antidepressants pharmacokinetics: a systematic review. Ann Gen Psychiatry. 2007;16:17. https://doi.org/10.1186/s12991-017-0140-8.

8. Schwartz JB. The Influence of Sex on Pharmacokinetics. Clin Pharmacokinet. 2003;42(2):107-21. https://doi.org/10.2165/00003088-200342020-00001.

9. Soldin OP, Mattison DR. Sex differences in pharmacokinetics and pharmacodynamics. Clin Pharmacokinet. 2009;48(3):143-57. https://doi.org/10.2165/00003088-200948030-00001. 
10. Unterecker S, Riederer P, Proft F, Maloney J, Deckert J, Pfuhlmann B. Effects of gender and age on serum concentrations of antidepressants under naturalistic conditions. J Neural Transm. 2013;120(8):1237-46. https://doi.org/10.1007/s00702-012-0952-2.

11. Castberg I, Westin AA, Skogvoll E, Spigset O. Effects of age and gender on the serum levels of clozapine, olanzapine, risperidone, and quetiapine. Acta Psychiatr Scand. 2017;136:455-64. https://doi.org/10.1111/acps.12794.

12. Hansen MR, Kuhlmann IB, Pottegard A, Damkier P. Therapeutic Drug Monitoring of Venlafaxine in an Everyday Clinical Setting: Analysis of Age, Sex and Dose Concentration Relationships. Basic Clin Pharmacol Toxicol. 2017;121(4):298-302. https://doi.org/10.1111/bcpt.12796.

13. Sigurdsson HP, Hefner G, Ben-Omar N, Köstlbacher A, Wenzel-Seifert K, Hiemke C. Steady-state serum concentrations of venlafaxine in patients with late-life depression. Impact of age, sex and BMI. J Neural Transm. 2015;122(5):721-9. https://doi.org/10.1007/s00702-014-1317-9.

14. Unterecker S, Hiemke C, Greiner C, Haen E, Jabs B, Deckert J. The effect of age, sex, smoking and comedication on serum levels of venlafaxine and O-desmethylvenlafaxine under naturalistic conditions. Pharmacopsychiatry. 2012;45(6):229-35. https://doi.org/10.1055/s-0031-1301366.

15. Scherf-Clavel M, Deckert J, Menke A, Unterecker S. Smoking Is Associated With Lower Dose-Corrected Serum Concentrations of Escitalopram. J Clin Psychopharmacol. 2019;39(5):485-8. https://doi.org/10.1097/JCP.0000000000001080.

16. Lobo ED, Quinlan T, O’Brien L, Knadler MP, Heathman M. Population pharmacokinetics of orally administered duloxetine in patients: implications for dosing recommendation. Clin Pharmacokinet. 2009;48(3):189-97. https://doi.org/10.2165/00003088-200948030-00005.

17. D'Incau P, Lapeyre-Mestre M., Carvajal A, Donati M, Salado I, Rodriguez L. No differences between men and women in adverse drug reactions related to psychotropic drugs: a survey from France, Italy and Spain. Fundam Clin Pharmacol. 2014;28(3):342-8. https://doi.org/10.1111/fcp.12032.

18. Greenblatt DJ, Harmatz JS, Roth T. Zolpidem and Gender: Are Women Really At Risk? J Clin Psychopharmacol. 2019;39(3):189-99. https://doi.org/10.1097/JCP.0000000000001026.

19. Zopf Y, Rabe C, Neubert A, Gassmann KG, Rascher W, Hahn EG. Women encounter ADRs more often than do men. Eur J Clin Pharmacol. 2008;64(10):999-1004. https://doi.org/10.1007/s00228-0080494-6.

20. Greil W, Zhang X, Stassen H, Grohmann R, Bridler R, Hasler G. Cutaneous adverse drug reactions to psychotropic drugs and their risk factors - a case-control study. Eur Neuropsychopharmacol. 2019;29(1):111-21. https://doi.org/10.1016/j.euroneuro.2018.10.010.

21. Beijer HJ, de Blaey CJ. Hospitalisations caused by adverse drug reactions (ADR): a meta-analysis of observational studies. Pharm World Sci. 2002;24(2):46-54. https://doi.org/10.1023/a:1015570104121.

22. Gray MP, Dziuba G, Quach K, Wong A, Smithburger PL, Seybert AL. Assessing Adverse Drug Reactions from Psychotropic Medications Reported to the U.S. Food and Drug Administration in Older Adults. Am J Geriatr Psychiatry. 2019;27(2):181-5. https://doi.org/10.1016/j.jagp.2018.09.013. 
23. Singh H, Yacob M, Sabu L, K Mamatha. Adverse drug reactions monitoring of psychotropic drugs: a tertiary care centre study. Open J of Psychiatry \& Allie Sci. 2017;8(2):136-40. https://doi.org/10.5958/2394-2061.2017.00009.X.

24. Bakken GV, Rudberg I, Molden E, Refsum H, Hermann M. Pharmacokinetic variability of quetiapine and the active metabolite N-desalkylquetiapine in psychiatric patients. Ther Drug Monit. 2011;33(2):222-6. https://doi.org/10.1097/FTD.0b013e31821160c4.

25. De Mendonça Lima CA, Baumann P, Brawand-Amey M, Brogli C, Jacquet S, Cochard N. Effect of age and gender on citalopram and desmethylcitalopram steady-state plasma concentrations in adults and elderly depressed patients. Prog Neuropsychopharmacol Biol Psychiatry. 2005;29(6):952-6. https://doi.org/10.1016/j.pnpbp.2005.06.001.

26. Gaillot J, Le Roux Y, Houghton GW, Dreyfus JF. Critical Factors for Pharmacokinetics of Zopiclone in the Elderly and in Patients with Liver and Renal Insufficiency. Sleep. 1987;10:7-21. https://doi.org/10.1093/sleep/10.suppl_1.7.

27. Hiemke C, Bergemann N, Clement HW, Conca A, Deckert J, Domschke K. Consensus Guidelines for Therapeutic Drug Monitoring in Neuropsychopharmacology: Update 2017. Pharmacopsychiatry. 2018;51(1-02):9-62. https://doi.org/10.1055/s-0043-116492.

28. KatzmanMA, Bleau P, Blier P, Chokka P, Kjernisted K, Van Ameringen M. Canadian clinical practice guidelines for the management of anxiety, posttraumatic stress and obsessive-compulsive disorders. BMC Psychiatry. 2014;14:S1. https://doi.org/10.1186/1471-244X-14-S1-S1.

29. Tveit K, Hermann M, Birkeland Waade R, Miodini Nilsen R, Wallerstedt SM, Molden E. Use of Antidepressants in Older People during a 10-Year Period: An Observational Study on Prescribed Doses and Serum Levels. Drugs Aging. 2020;37(9):691-701. https://doi.org/10.1007/s40266-02000784-9. 29a. Petra A Thürmann. Pharmacodynamics and pharmacokinetics in older adults. Curr Opin Anaesthesiol 2020; 33(1):109-113.doi: 10.1097/AC0.0000000000000814.

30. Glaeske G, Heitmann CG, Höfel F, Schicktanz C. „Gender-Specific Drug Prescription in Germany” Results from Prescriptions Analyses. In: Regitz-Zagrosek V, editor. Sex and Gender Differences in Pharmacology. Berlin Heidelberg : Springer; 2012. p. 149-167.

31. Liu KA, Mager NA. Women's involvement in clinical trials: historical perspective and future implications. Pharm Pract (Granada). 2016;14(1):708. http://doi.org/10.18549/PharmPract.2016.01.708.

32. Sørup FKH, Eriksson R, Westergaard D, Hallas J, Brunak S, Andersen SE. Sex differences in textmined possible adverse drug events associated with drugs for psychosis. J Psychopharmacol. 2020;34(5):532-9. https://doi.org/10.1177/0269881120903466.

33. FDA, U.S. Food and Drug Administration. Risk of next-day impairment after use of insomnia drugs; FDA requires lower recommended doses for certain drugs containing zolpidem (Ambien, Ambien CR, Edular, and Zolpimist). Rockville, MD; 2013. 
34. FDA U.S. Food and Drug Administration. FDA approves new label changes and dosing for zolpidem products and a recommendation to avoid driving the day after using Ambien CR. 2013. https://www.fda.gov/drugs/drug-safety-and-availability/fda-drug-safety-communication-fdaapproves-new-label-changes-and-dosing-zolpidem-products-and/. Accessed August 2020.

35. Engel RR, Grohmann R, Ruther E, Hippius H. Research Methods in Drug Surveillance. Pharmacopsychiatry. 2004;37:12-5. https://doi.org/10.1055/s-2004-815506.

36. Grohmann R, Engel RR, Moller HJ, Ruther E, van der Velden JW, Stubner S. Flupentixol use and adverse reactions in comparison with other common first- and second-generation antipsychotics: data from the AMSP study. Eur Arch Psychiatry Clin Neurosci. 2014;264(2):131-41. https://doi.org/10.1007/s00406-013-0419-y.

37. Grohmann R, Engel RR, Ruther E, Hippius H. The AMSP drug safety program: methods and global results. Pharmacopsychiatry. 2004;37:4-11. https://doi.org/10.1055/s-2004-815505.

38. Olubodun JO, Ochs HR, von Moltke LL, Roubenoff R, Hesse LM, Harmatz JS. Pharmacokinetic properties of zolpidem in elderly and young adults: possible modulation by testosterone in men. $\mathrm{Br} \mathrm{J}$ Clin Pharmacol. 2003;56(3):297-304. https://doi.org/10.1046/j.0306-5251.2003.01852.x.

39. Gaillot J, Heusse D, Hougton GW, Marc Aurele J, Dreyfus JF. Pharmacokinetics and metabolism of zopiclone. Pharmacology. 1983;27:76-91. https://doi.org/10.1159/000137914.

40. Greenblatt DJ, Allen MD, Locniskar A, Harmatz JS, Shader RI. Lorazepam kinetics in the elderly. Clin Pharmacol Ther. 1979;26(1):103-13. https://doi.org/10.1002/cpt1979261103.

41. Federal Statistical Office of Germany (statistisches Bundesamt). Mikrozensus 2017 - Fragen zur Gesundheit. 2018. https://www.destatis.de/DE/Themen/GesellschaftUmwelt/Gesundheit/Gesundheitszustand-Relevantes-Verhalten/Publikationen/DownloadsGesundheitszustand/koerpermasse-5239003179004.pdf?_blob=publicationFile. Accessed October 2020.

\section{Figures}




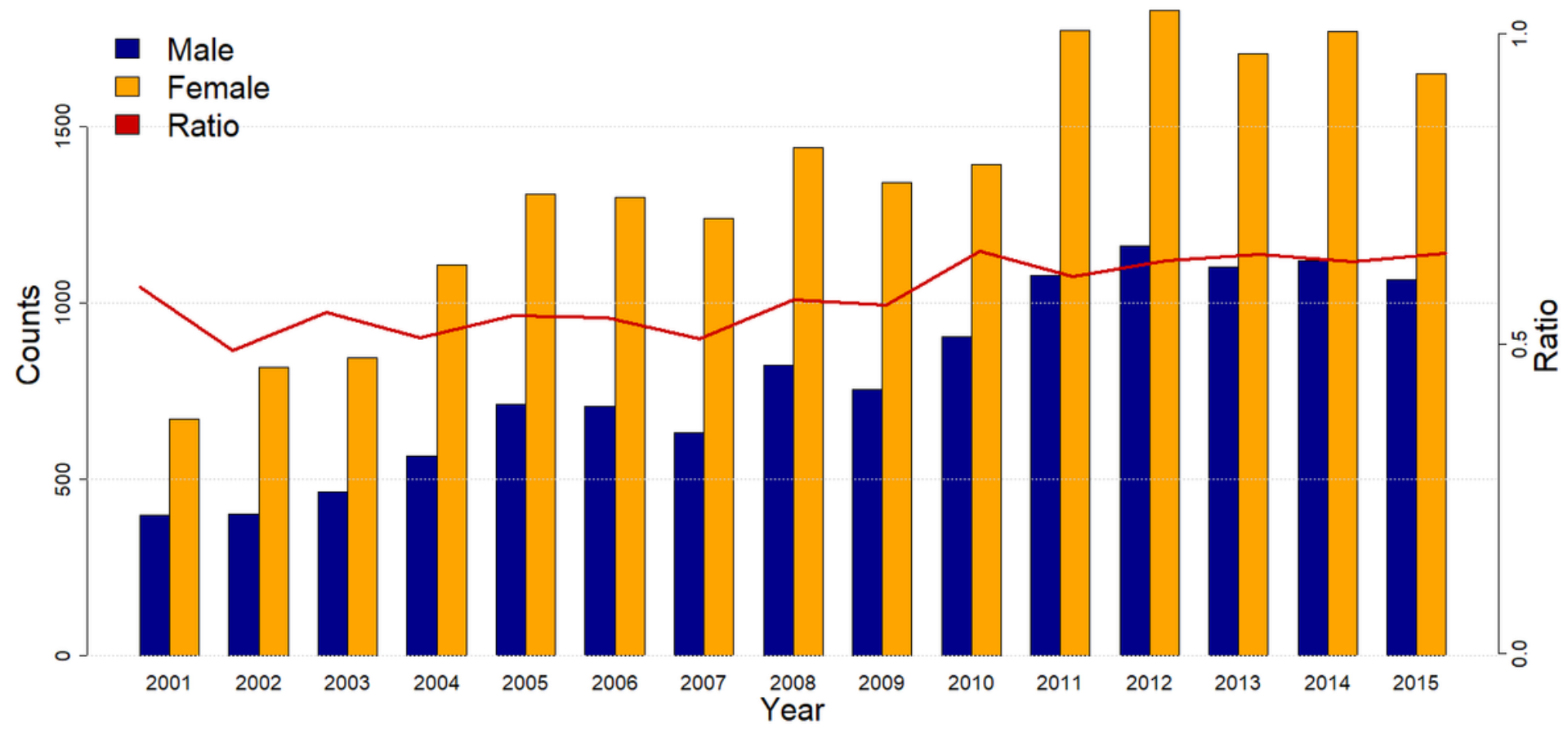

Figure 1

Number of patients with MDD by year Number of male and female patients in our sample by calendar years between 2001 and 2015. Blue: Males. Orange: Females. Red: males divided by females. The ratio between male and female changes only slightly along the years. MDD: Major Depressive Disorders

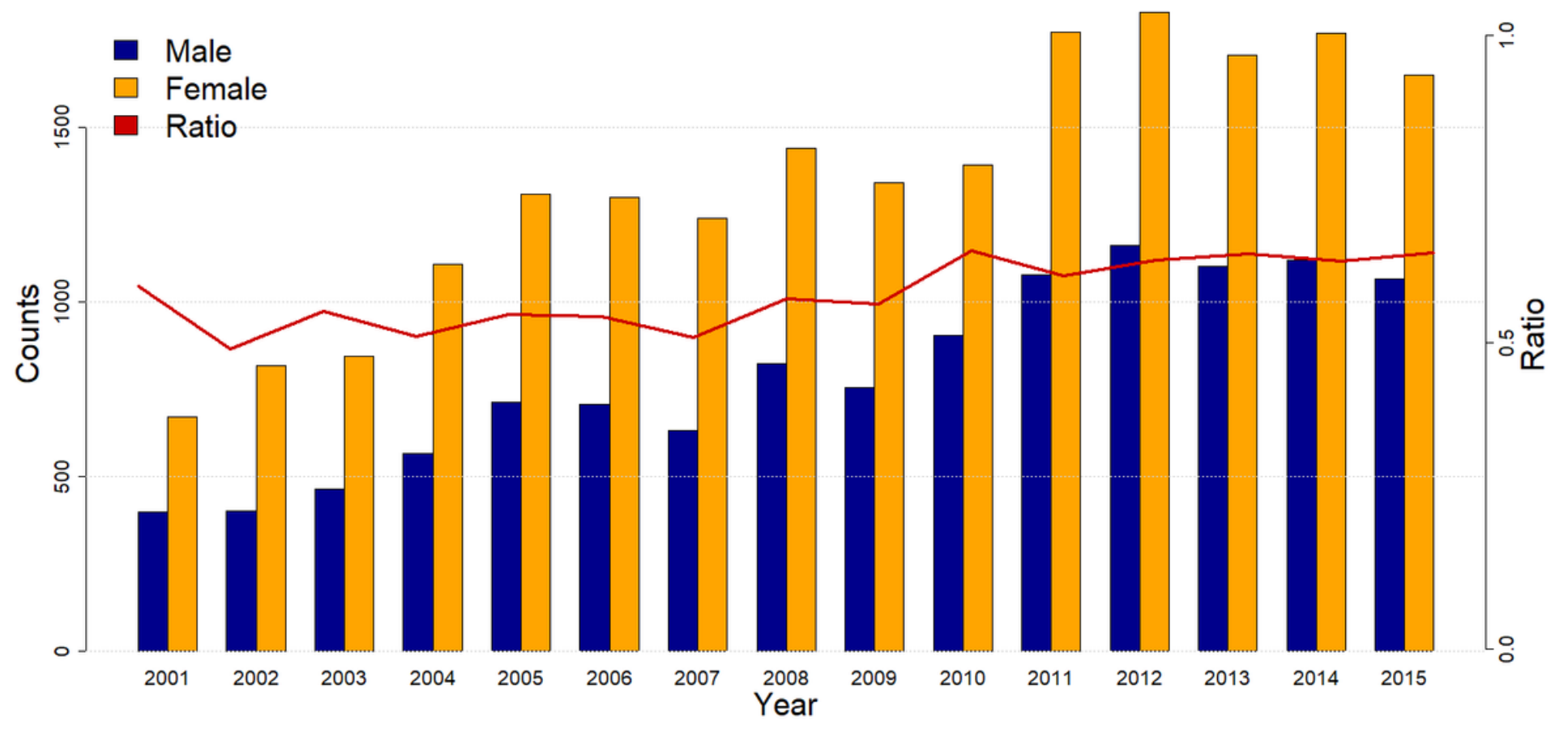

Figure 1 
Number of patients with MDD by year Number of male and female patients in our sample by calendar years between 2001 and 2015. Blue: Males. Orange: Females. Red: males divided by females. The ratio between male and female changes only slightly along the years. MDD: Major Depressive Disorders

\section{Female}
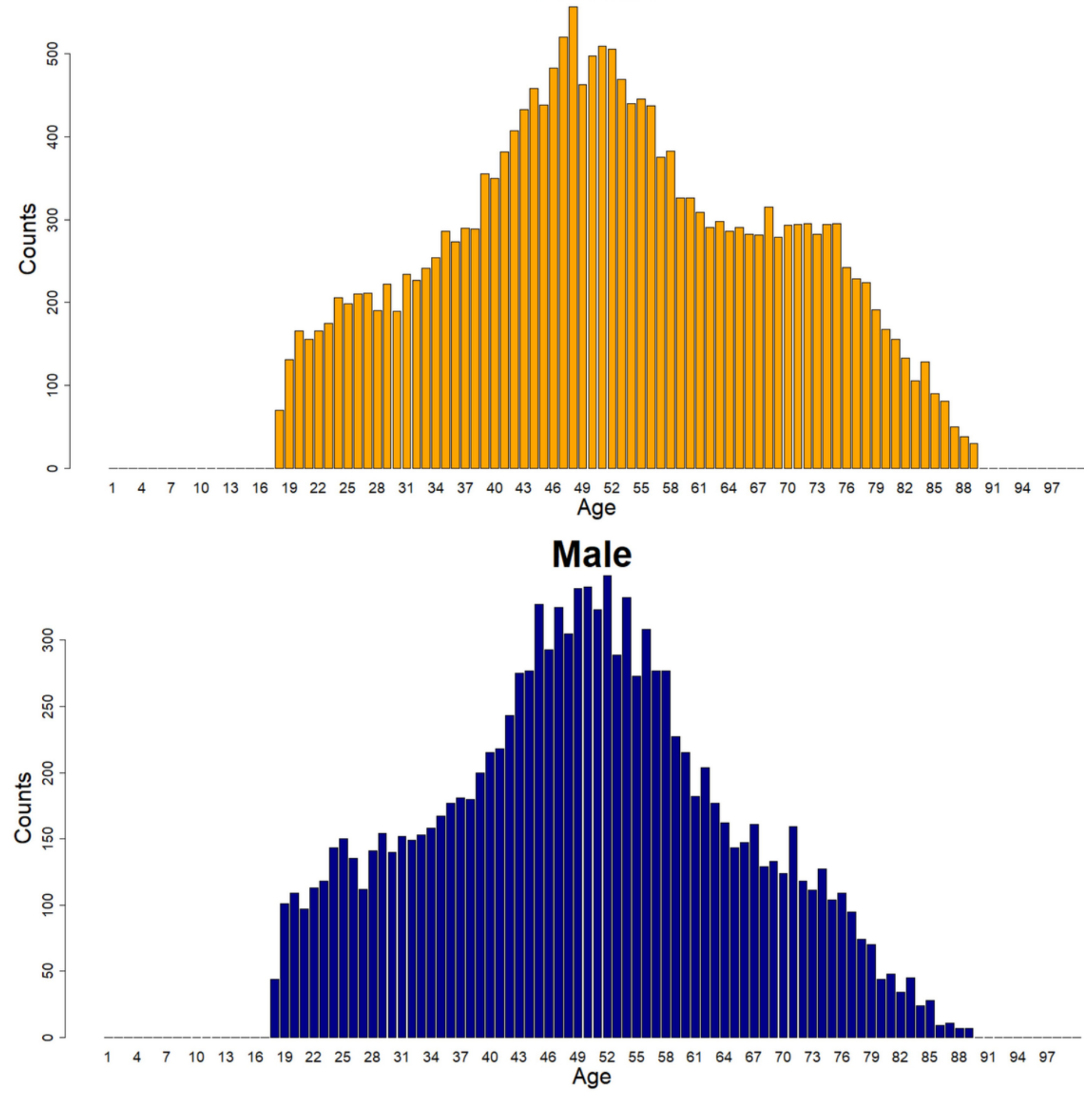

Figure 2

Age distributions On the top the female age distribution, on the bottom the male age distribution. In our sample are proportionately more elderly women then elderly men, and overall more women. 


\section{Female}
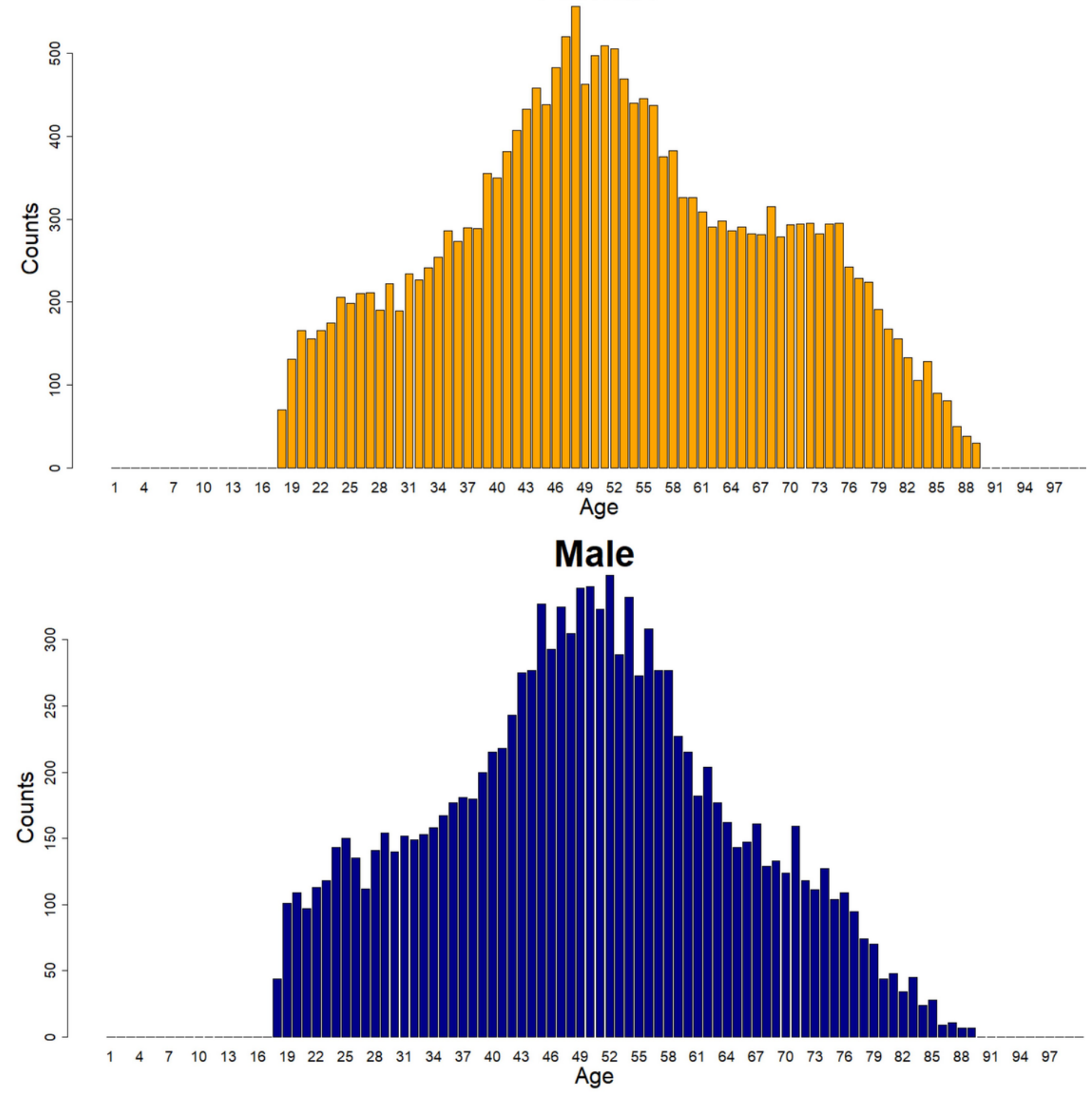

Figure 2

Age distributions On the top the female age distribution, on the bottom the male age distribution. In our sample are proportionately more elderly women then elderly men, and overall more women. 


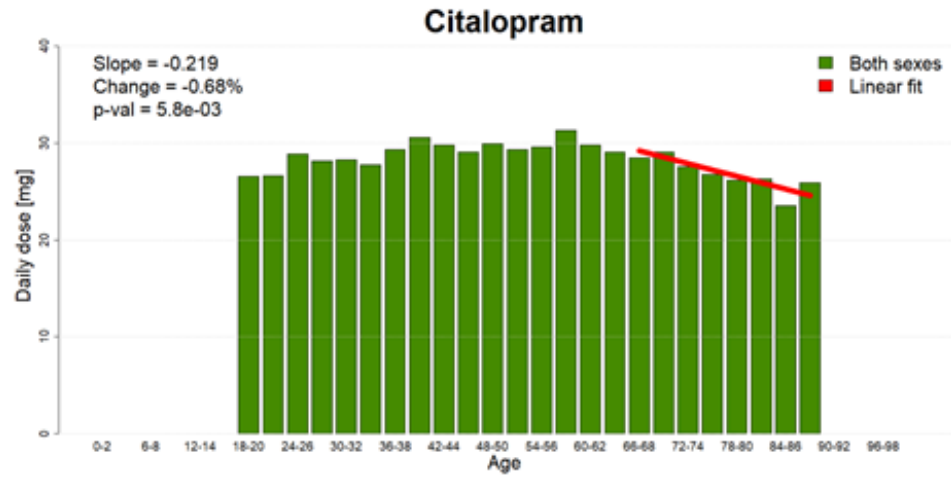

(a) Citalopram

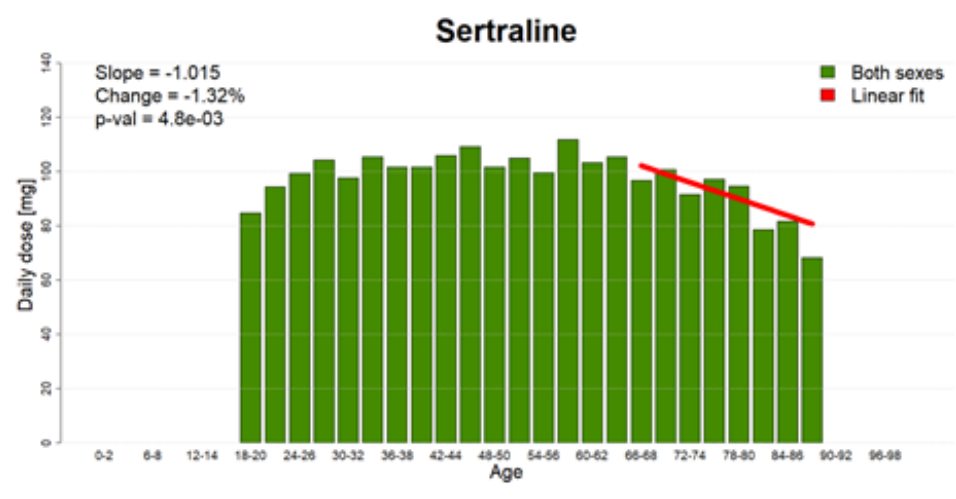

(c) Sertraline

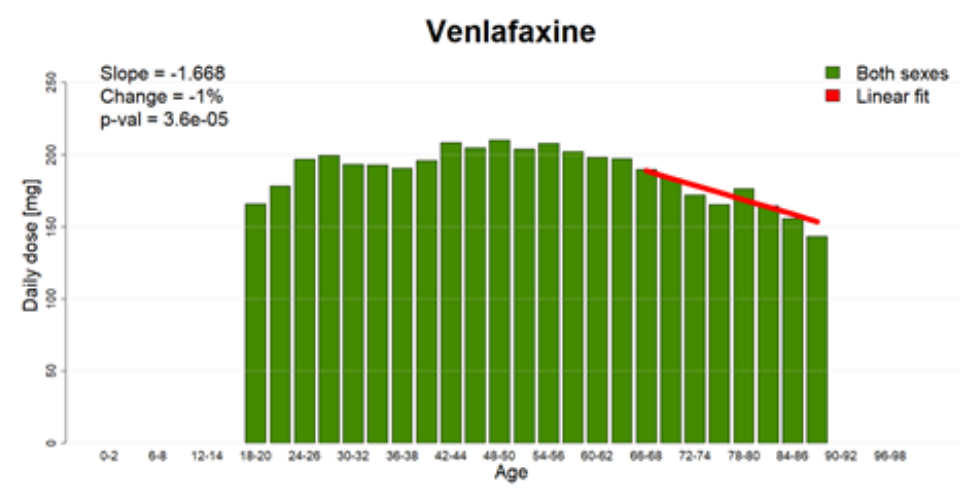

(e) Venlafaxine

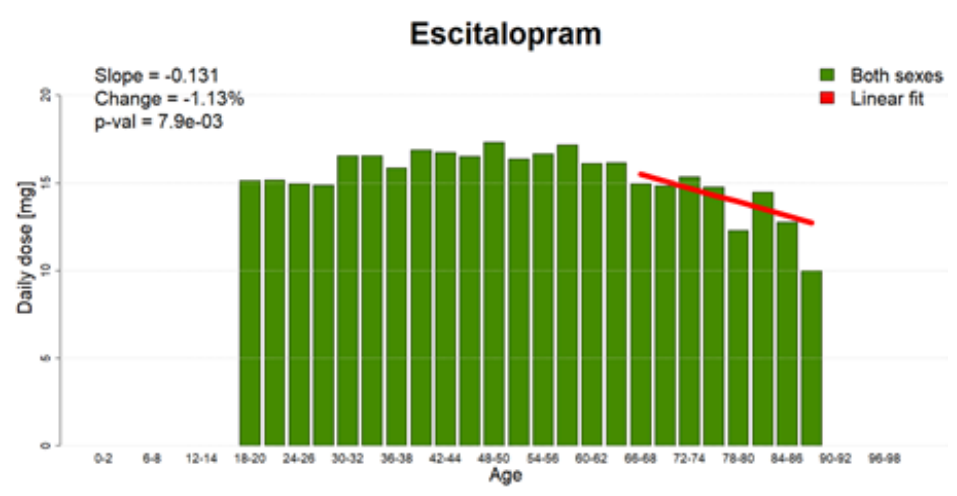

(b) Escitalopram

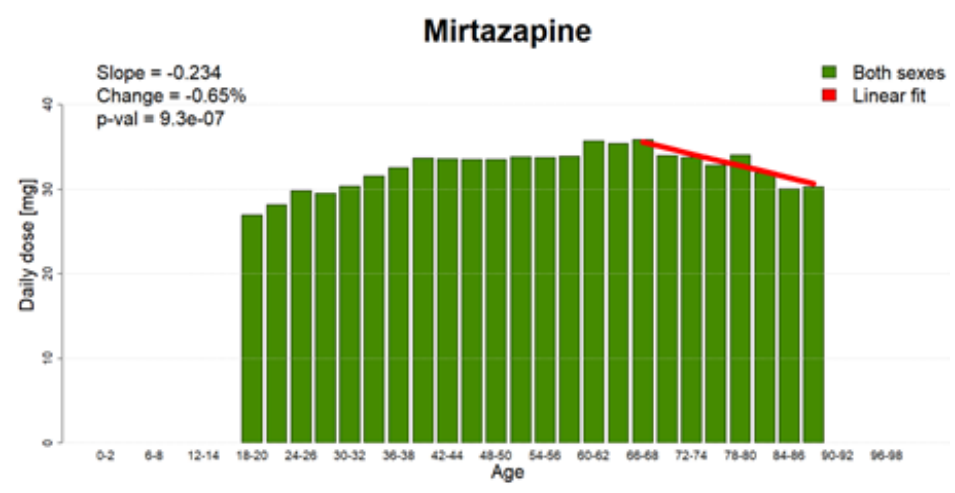

(d) Mirtazapine

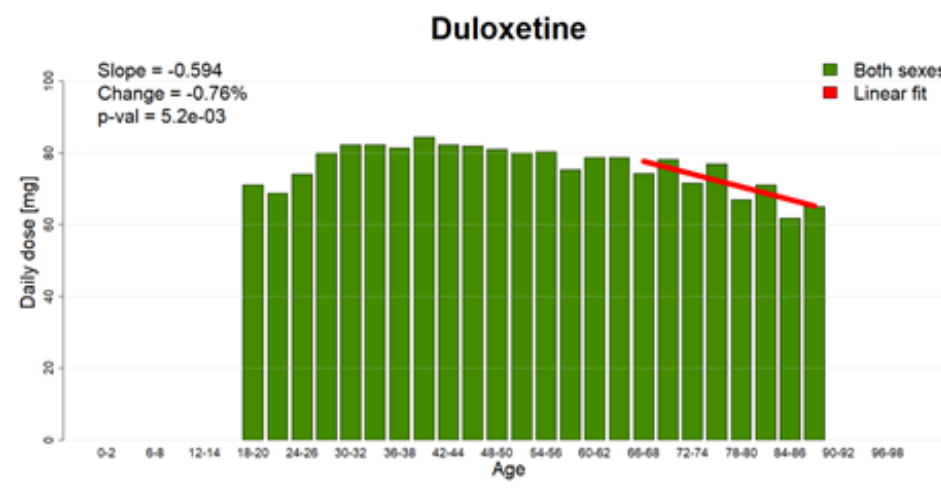

(f) Duloxetine

\section{Figure 3}

Daily dose by age: Antidepressants Daily prescribed doses (mg) of antidepressants by age including both male and female patients. The height of the bars corresponds to the mean daily dosage for inpatients within age groups of three consecutive years. The patients are between 18 and 89 years old. In red, a linear model fitted to patients between 66 and 89 years old is shown. Slope: The slope of the linear model, describing the average absolute difference of daily dose in relation to the previous year in elderly patients. Change: Average percentage change in relation to the previous year in elderly patients. p-val: $p$ value under the hypothesis that the slope is zero, $p<0.001$ in all cases. 


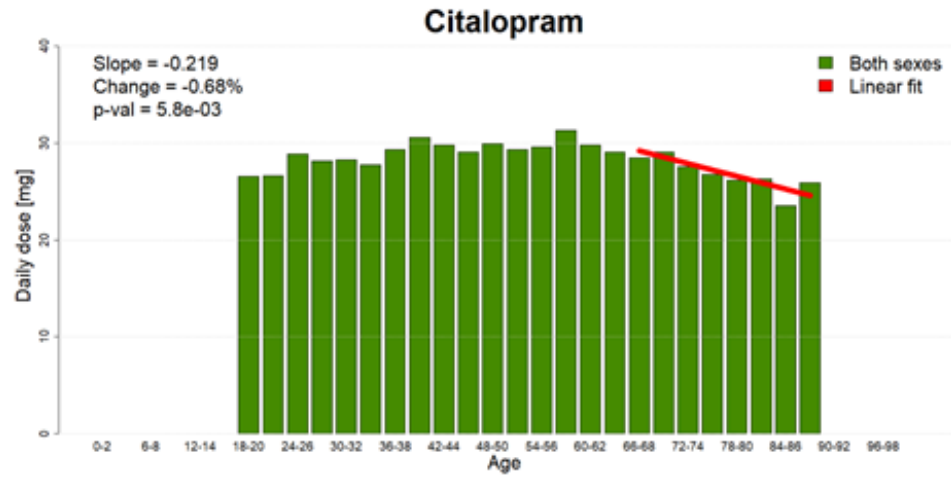

(a) Citalopram

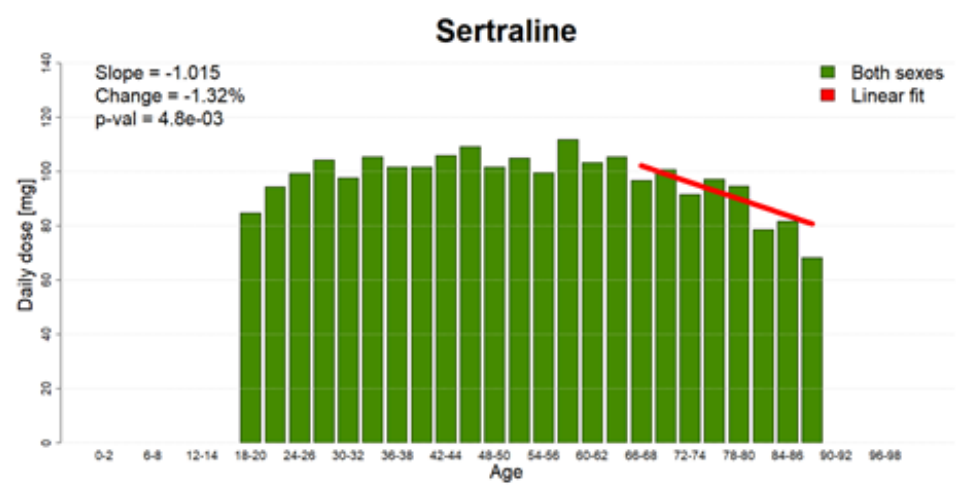

(c) Sertraline

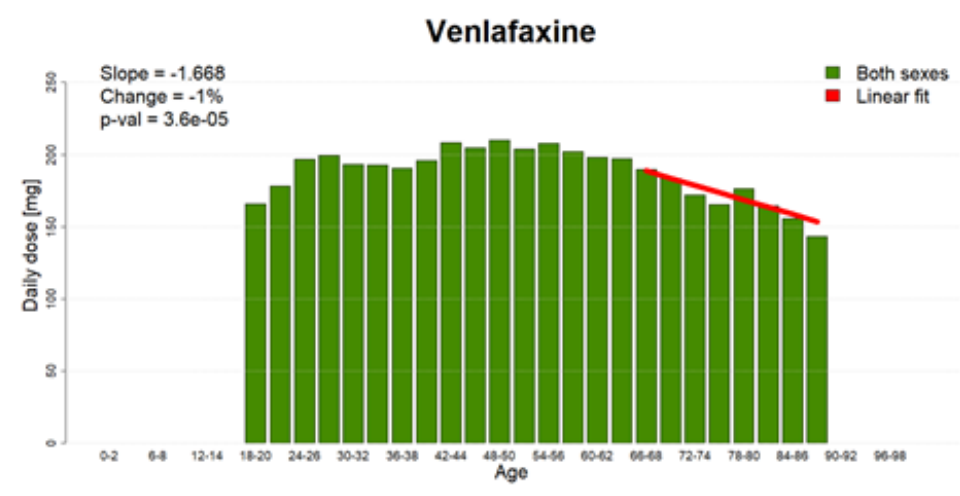

(e) Venlafaxine

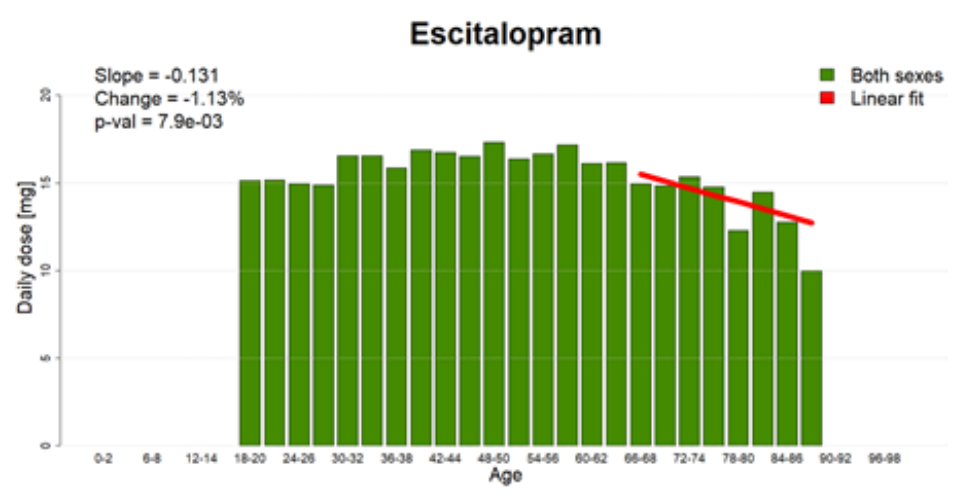

(b) Escitalopram

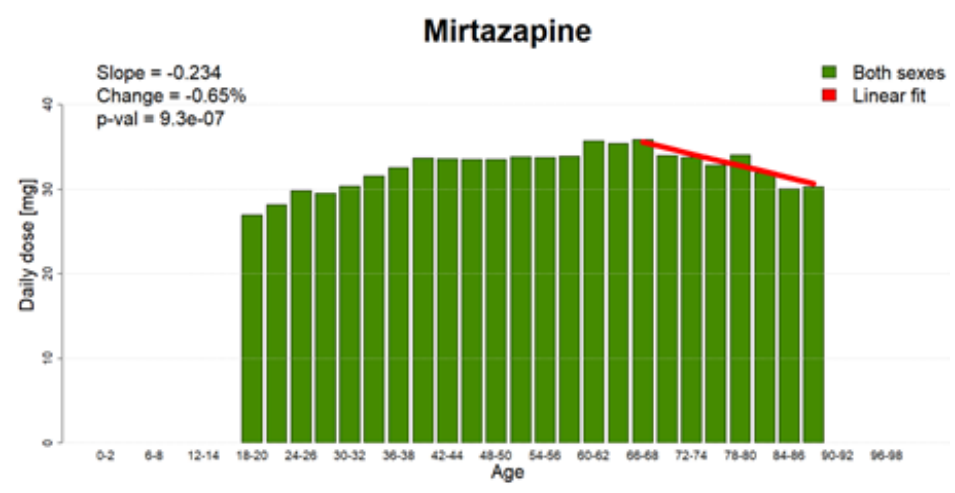

(d) Mirtazapine

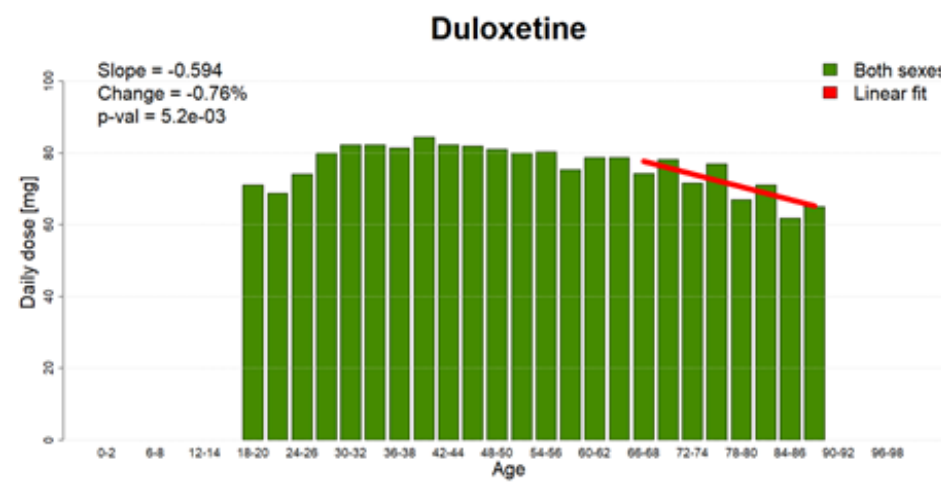

(f) Duloxetine

\section{Figure 3}

Daily dose by age: Antidepressants Daily prescribed doses (mg) of antidepressants by age including both male and female patients. The height of the bars corresponds to the mean daily dosage for inpatients within age groups of three consecutive years. The patients are between 18 and 89 years old. In red, a linear model fitted to patients between 66 and 89 years old is shown. Slope: The slope of the linear model, describing the average absolute difference of daily dose in relation to the previous year in elderly patients. Change: Average percentage change in relation to the previous year in elderly patients. p-val: $p$ value under the hypothesis that the slope is zero, $p<0.001$ in all cases. 


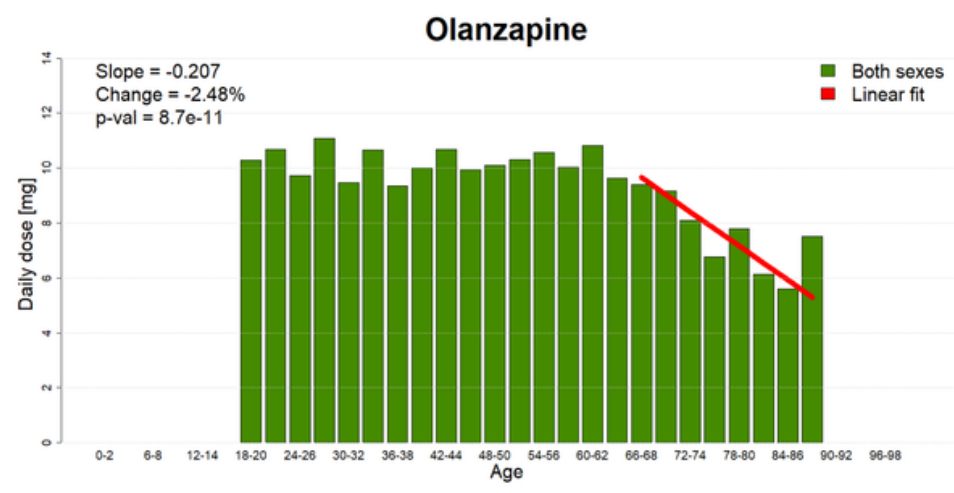

(a) Olanzapine

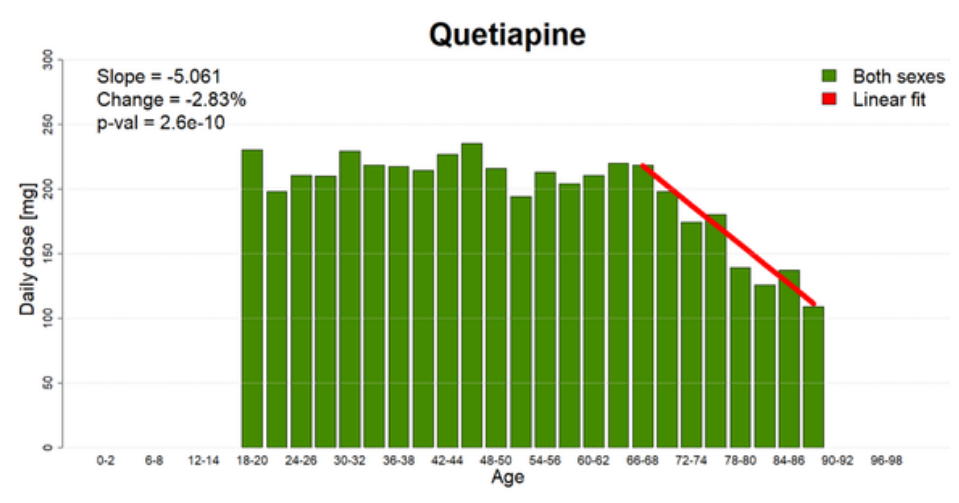

(b) Quetiapine

\section{Figure 4}

Daily dose by age: Antipsychotics Daily prescribed doses $(\mathrm{mg})$ of antipsychotics by age including both male and female patients. The height of the bars corresponds to the mean daily dosage for inpatients within age groups of three consecutive years. The patients are between 18 and 89 years old. In red, a linear model fitted to patients between 66 and 89 years old. Slope: The slope of the linear model, describing the average absolute difference of daily dose in relation to the previous year in elderly patients. Change: Average percentage change in relation to the previous year in elderly patients. $p$-val: $p$-value under the hypothesis that the slope is zero, $p<0.001$ in both cases.

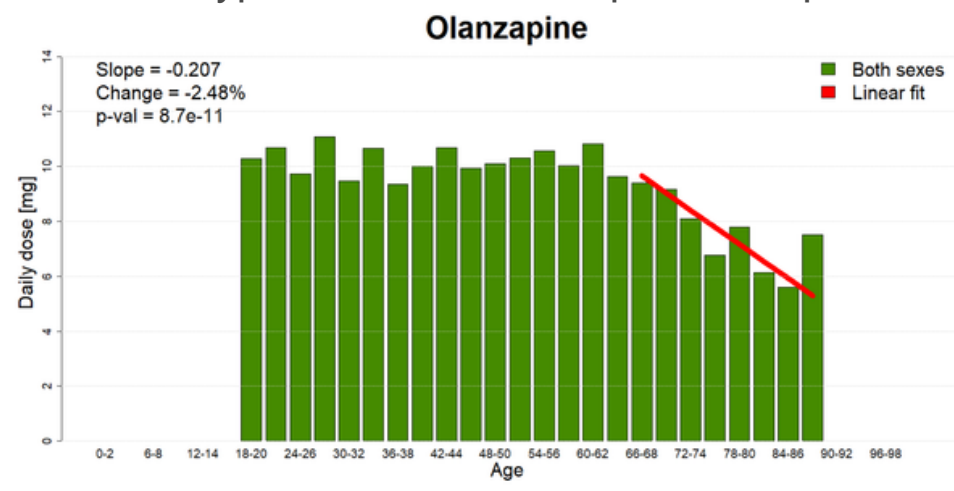

(a) Olanzapine

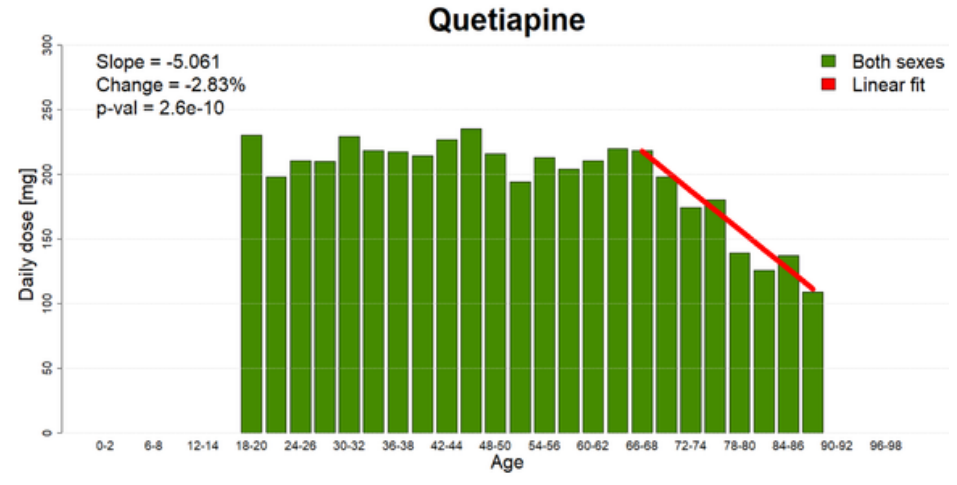

(b) Quetiapine

\section{Figure 4}

Daily dose by age: Antipsychotics Daily prescribed doses $(\mathrm{mg})$ of antipsychotics by age including both male and female patients. The height of the bars corresponds to the mean daily dosage for inpatients within age groups of three consecutive years. The patients are between 18 and 89 years old. In red, a linear model fitted to patients between 66 and 89 years old. Slope: The slope of the linear model, describing the average absolute difference of daily dose in relation to the previous year in elderly patients. Change: Average percentage change in relation to the previous year in elderly patients. $p$-val: $p$-value under the hypothesis that the slope is zero, $p<0.001$ in both cases. 
Zolpidem

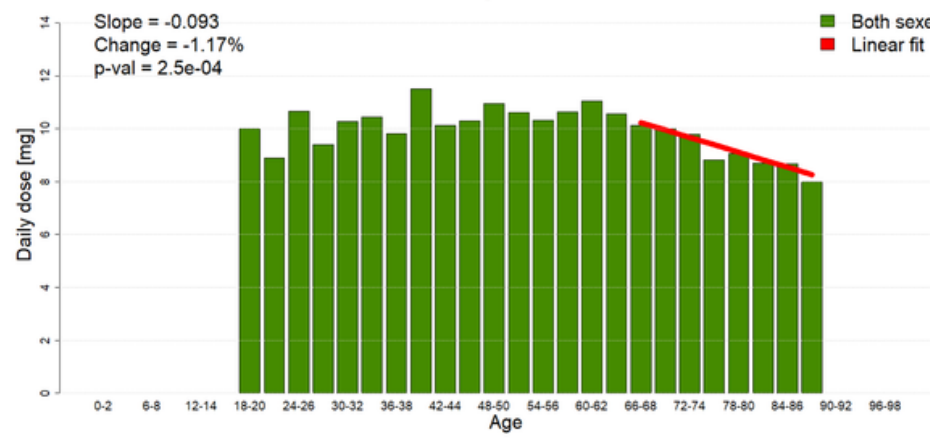

(a) Zolpidem

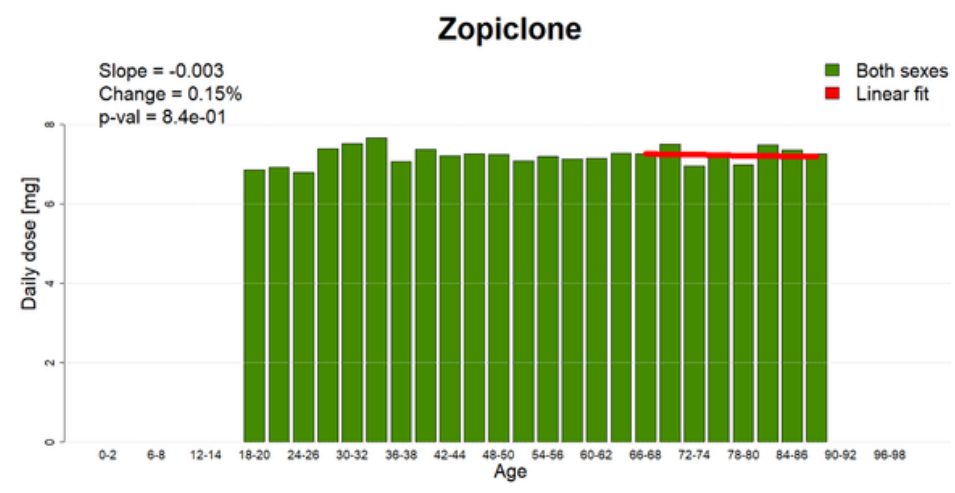

(b) Zopiclone

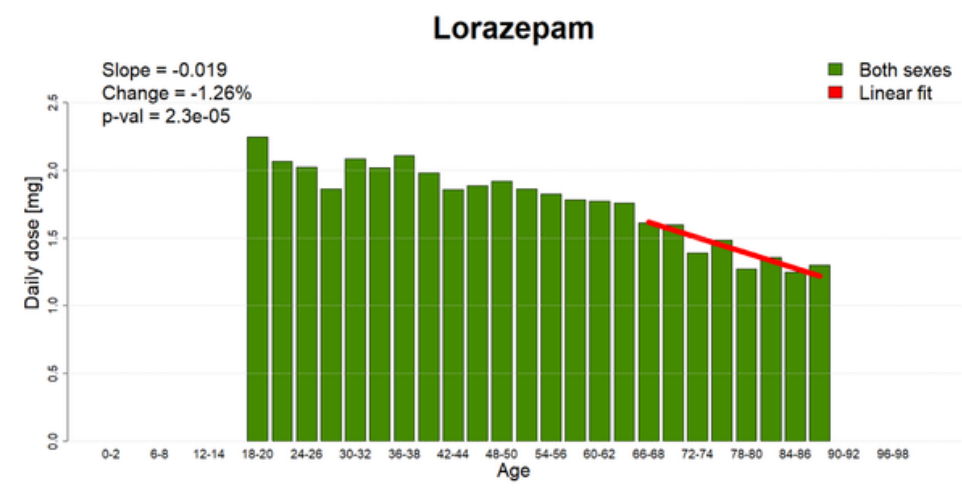

(c) Lorazepam

\section{Figure 5}

Daily dose by age: Hypnotics and Tranquilizers Daily prescribed doses (mg) of hypnotics and tranquilizers by age including both male a female patients. The height of the bars corresponds to the mean daily dosage for inpatients within age groups of three consecutive years. The patients are between 18 and 89 years old. In red, a linear model fitted to patients between 66 and 89 years old. Slope: The slope of the linear model, describing the average absolute difference of daily dose in relation to the previous year in elderly patients. Change: Average percentage change in relation to the previous year in elderly patients. p-val: p-value under the hypothesis that the slope is zero, n.s. (not significant) for zoplicone; $p<0.001$ in both other cases. 
Zolpidem

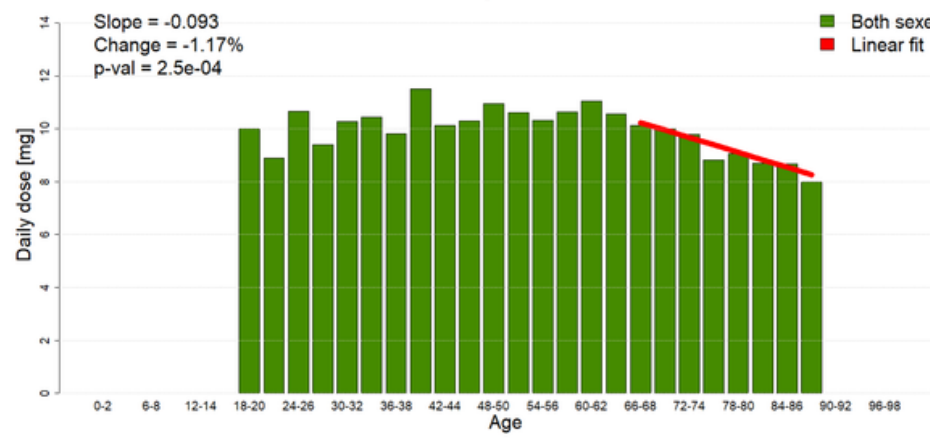

(a) Zolpidem

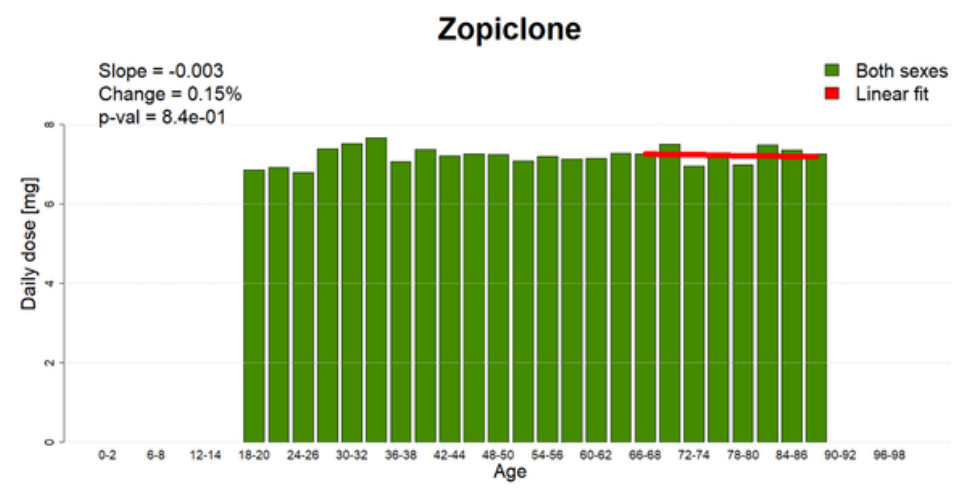

(b) Zopiclone

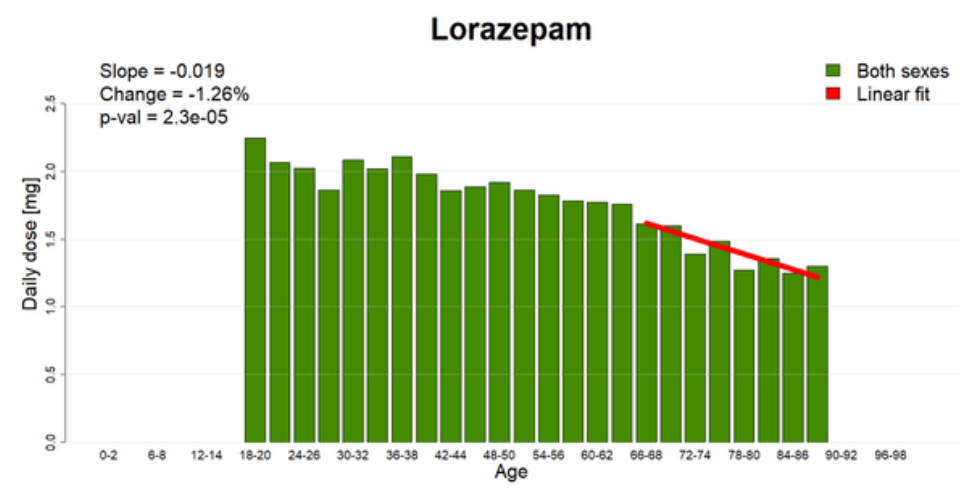

(c) Lorazepam

\section{Figure 5}

Daily dose by age: Hypnotics and Tranquilizers Daily prescribed doses $(\mathrm{mg})$ of hypnotics and tranquilizers by age including both male a female patients. The height of the bars corresponds to the mean daily dosage for inpatients within age groups of three consecutive years. The patients are between 18 and 89 years old. In red, a linear model fitted to patients between 66 and 89 years old. Slope: The slope of the linear model, describing the average absolute difference of daily dose in relation to the previous year in elderly patients. Change: Average percentage change in relation to the previous year in elderly patients. p-val: p-value under the hypothesis that the slope is zero, n.s. (not significant) for zoplicone; $p<0.001$ in both other cases. 


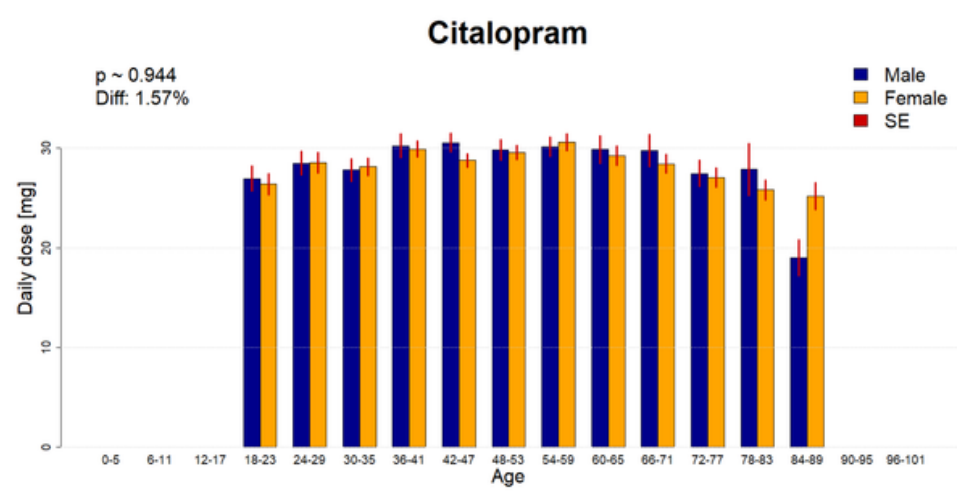

(a) Citalopram

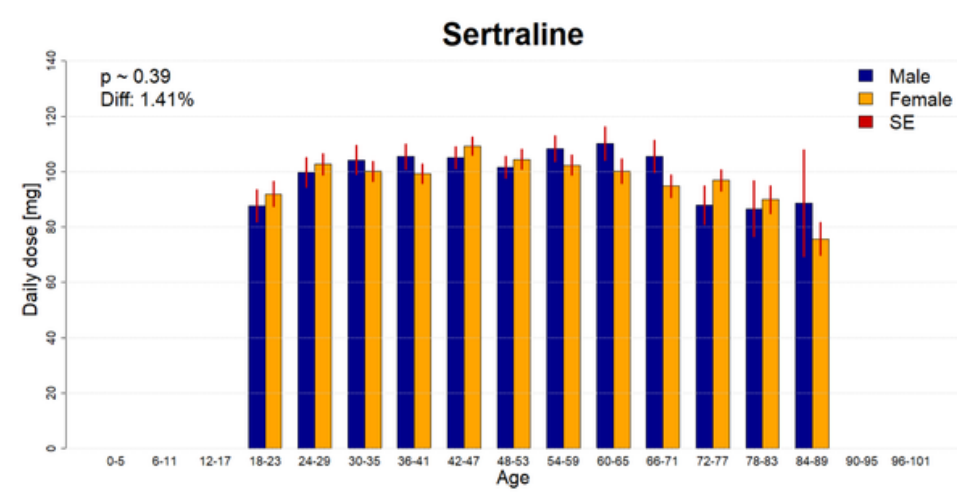

(c) Sertraline

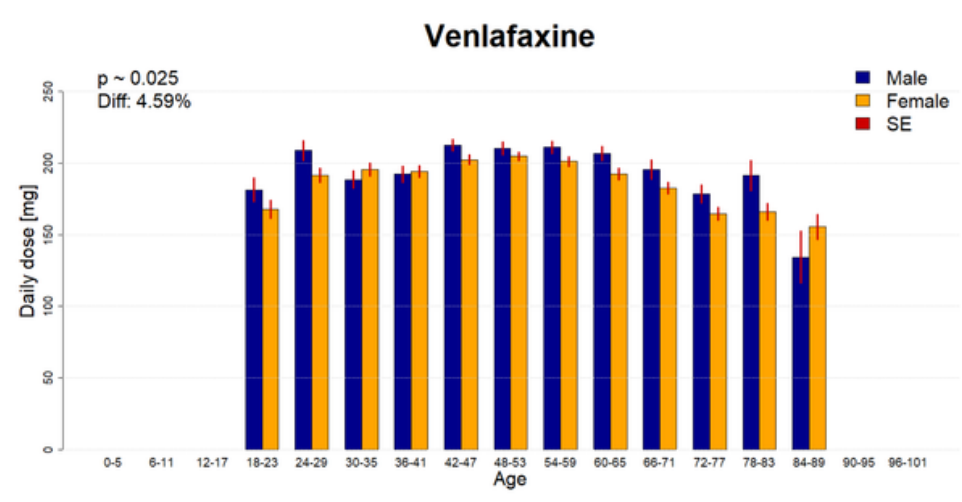

(e) Venlafaxine

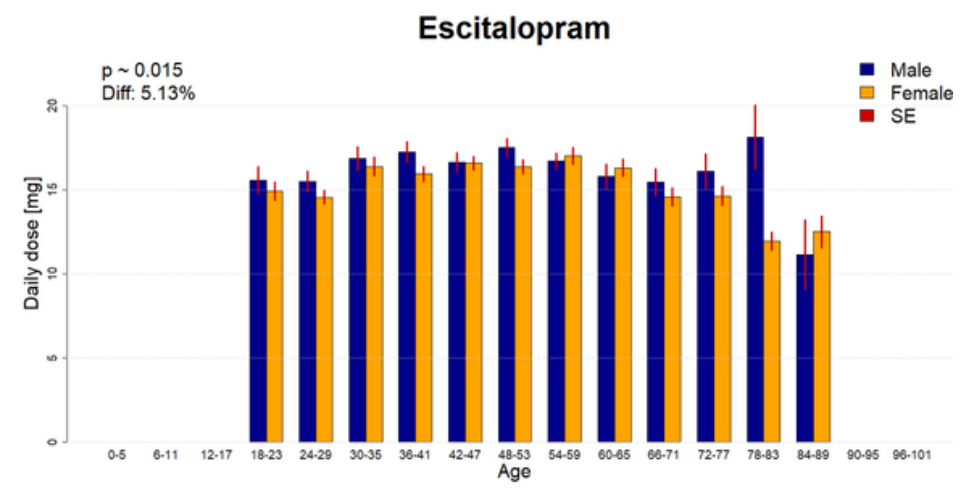

(b) Escitalopram

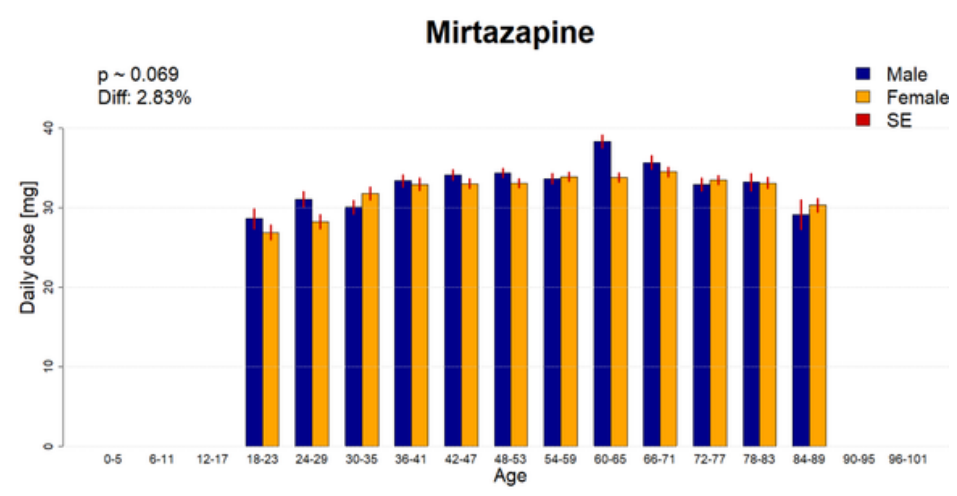

(d) Mirtazapine

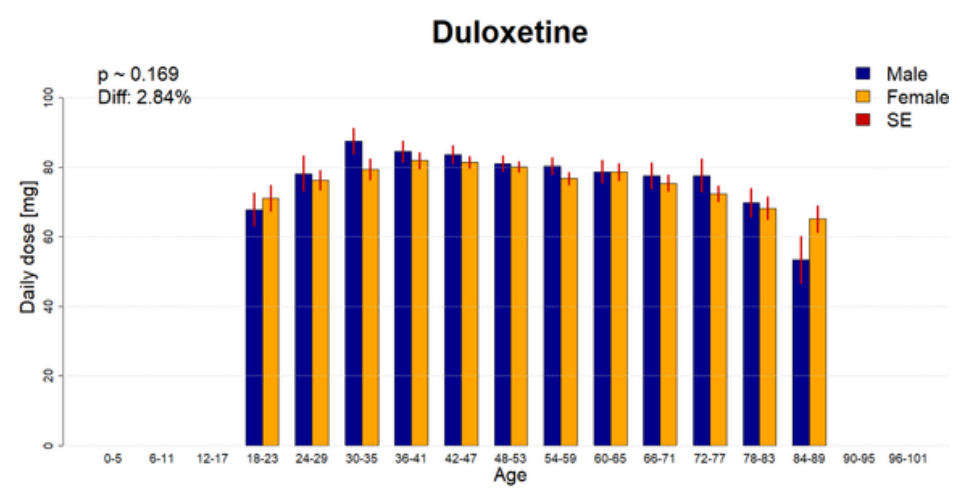

(f) Duloxetine

\section{Figure 6}

Daily dose by age and sex: Antidepressants Daily prescribed doses $(\mathrm{mg})$ of antidepressants by age: males in blue and female in orange. The standard error is depicted in red. The height of the bars corresponds to the mean daily dosage for inpatients within age groups of three consecutive years. The patients are between 18 and 89 years old. P-value: The $p$-value is computed from a (paired) Wilcoxon signed-rank test under the hypothesis that there are no differences between sexes: Diff: The percentage difference between sexes. Female patients are chosen as reference. From the plots can be seen, that the prescribed dosage differences between men and females are small. $p<0.05$ for escitalopram and venlafaxine; $n . s$ in all other cases. 


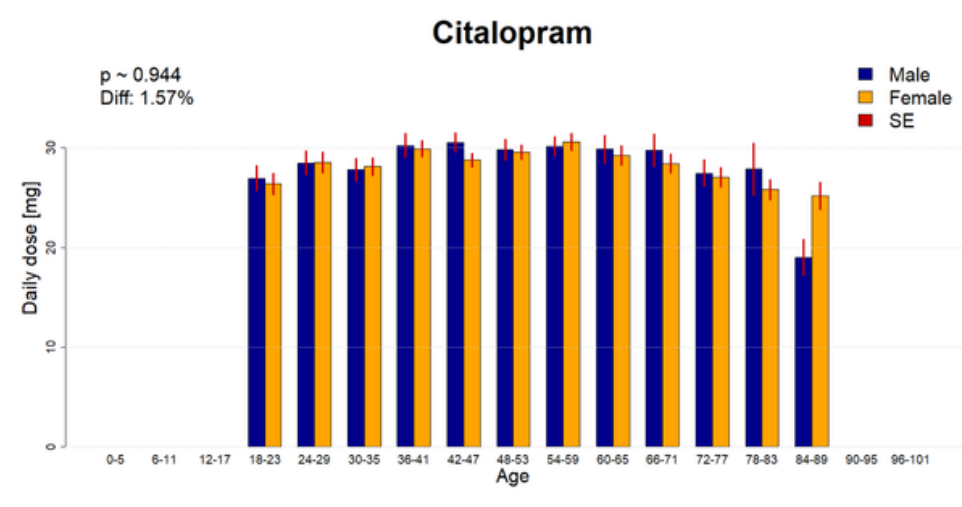

(a) Citalopram

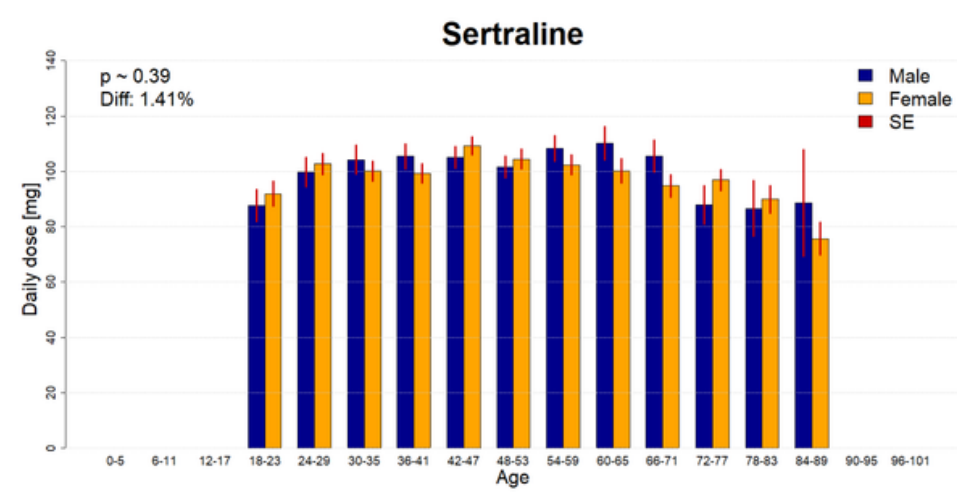

(c) Sertraline

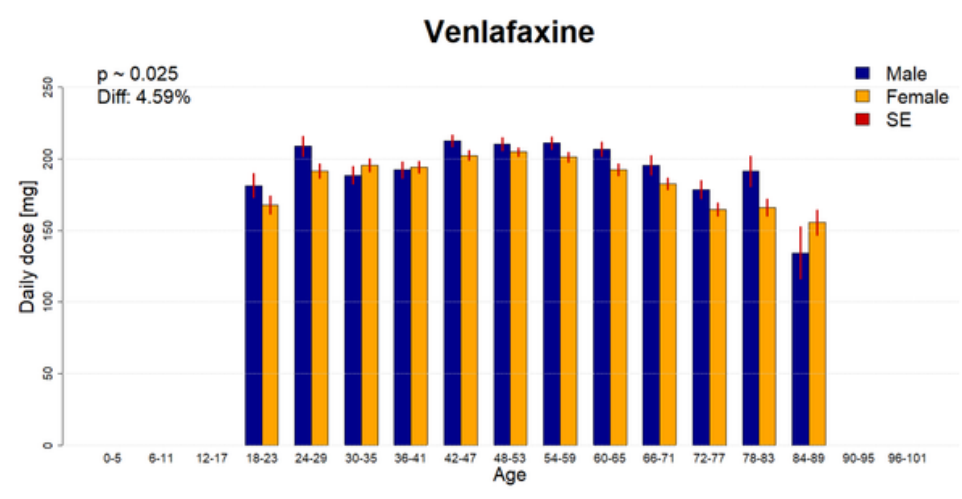

(e) Venlafaxine

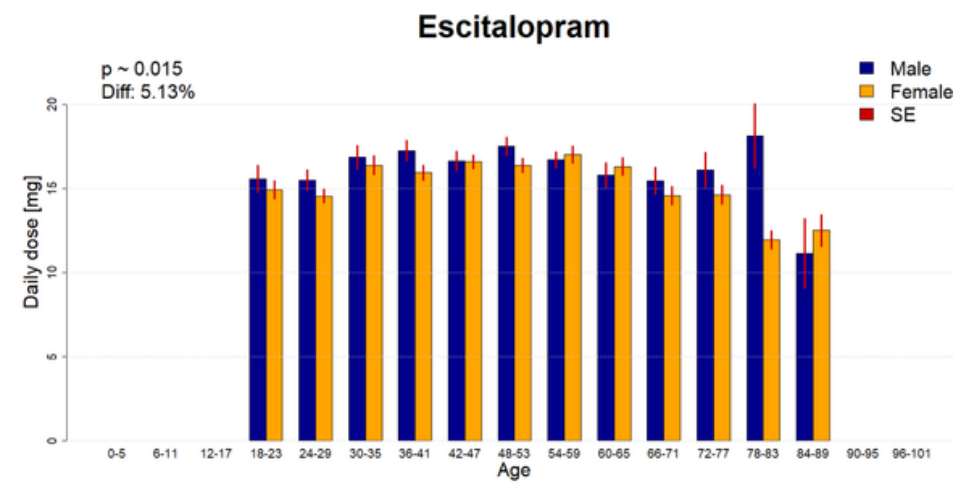

(b) Escitalopram

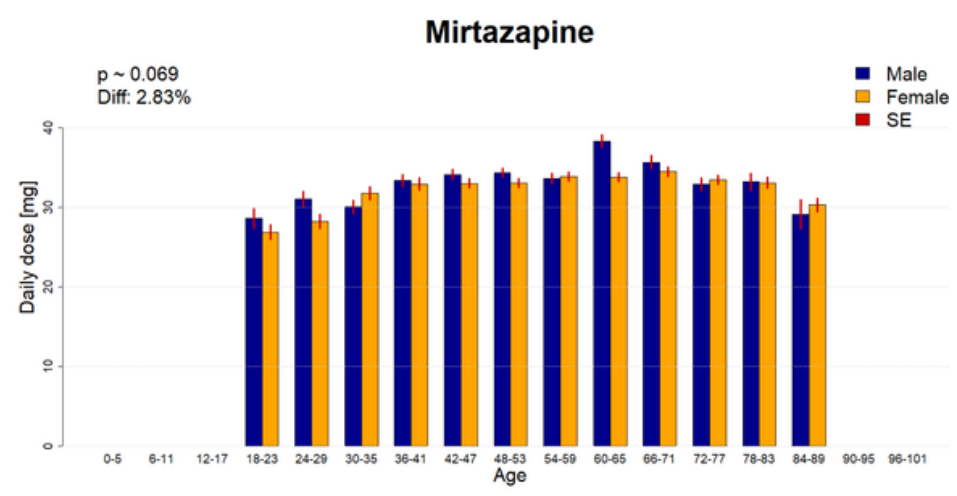

(d) Mirtazapine

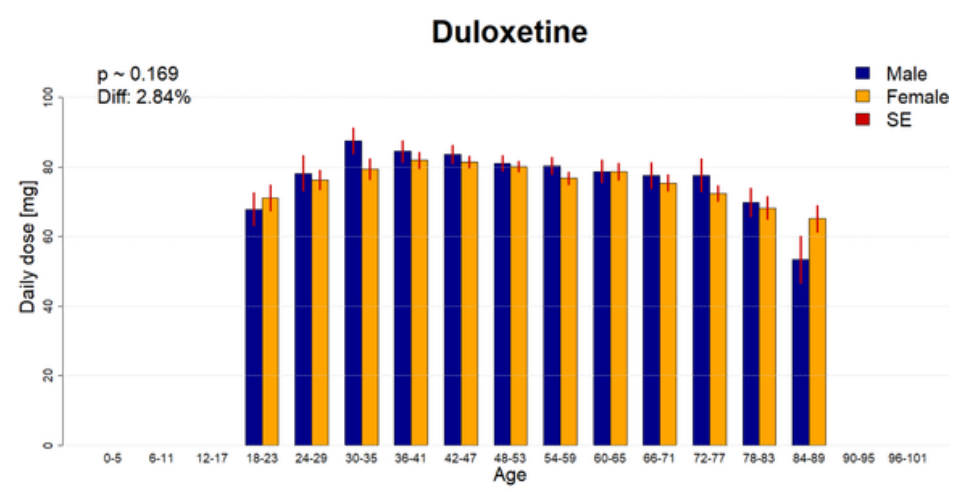

(f) Duloxetine

\section{Figure 6}

Daily dose by age and sex: Antidepressants Daily prescribed doses $(\mathrm{mg})$ of antidepressants by age: males in blue and female in orange. The standard error is depicted in red. The height of the bars corresponds to the mean daily dosage for inpatients within age groups of three consecutive years. The patients are between 18 and 89 years old. P-value: The $p$-value is computed from a (paired) Wilcoxon signed-rank test under the hypothesis that there are no differences between sexes: Diff: The percentage difference between sexes. Female patients are chosen as reference. From the plots can be seen, that the prescribed dosage differences between men and females are small. $p<0.05$ for escitalopram and venlafaxine; $n . s$ in all other cases. 


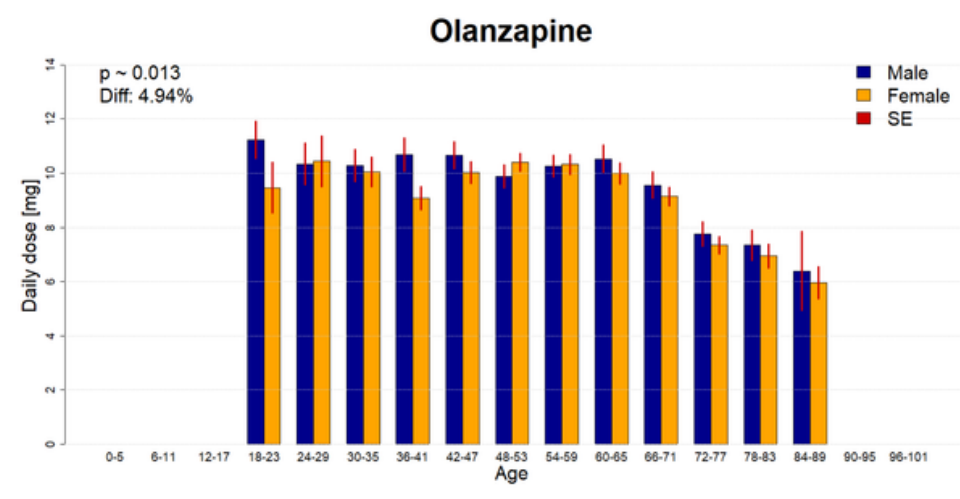

(a) Olanzapine

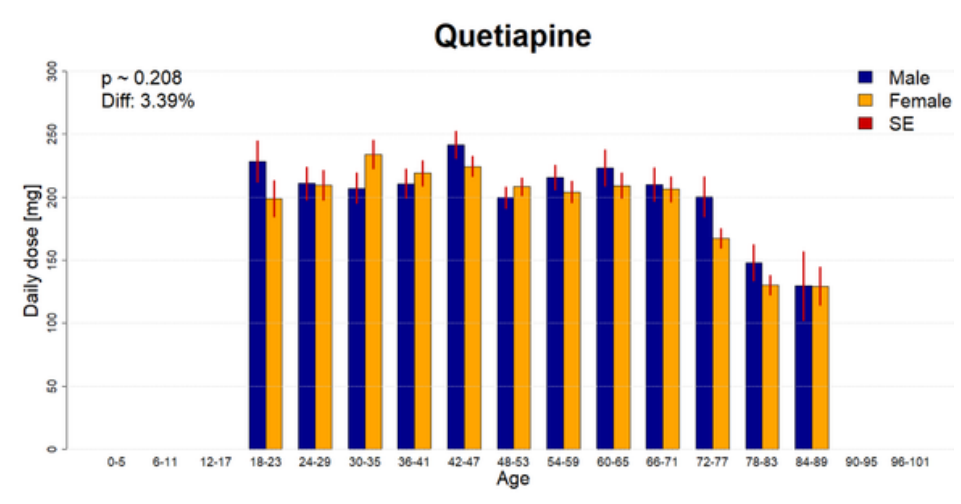

(b) Quetiapine

\section{Figure 7}

Daily dose by age and sex: Antipsychotics Daily prescribed doses $(\mathrm{mg})$ of antipsychotics by age: males in blue and female in orange. The standard error is depicted in red. The height of the bars corresponds to the mean daily dosage for inpatients within age groups of three consecutive years. The patients are between 18 and 89 years old. P-value: The p-value is computed from a (paired) Wilcoxon signed-rank test under the hypothesis that there are no differences between sexes. Diff: The percentage difference between sexes. Female patients are chosen as reference. From the plots can be seen, that the prescribed dosage differences between men and females are either small or statistically insignificant: $p<0.05$ for olanzapine, n.s. for quetiapine.

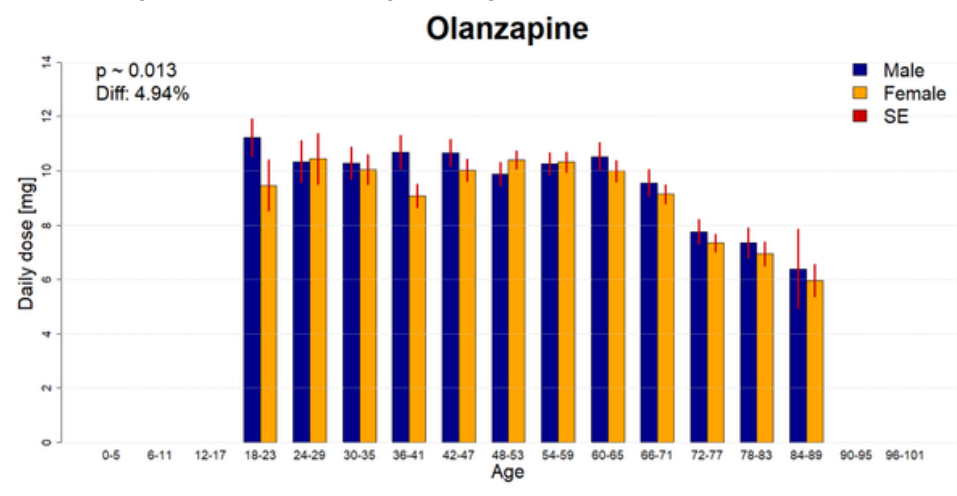

(a) Olanzapine

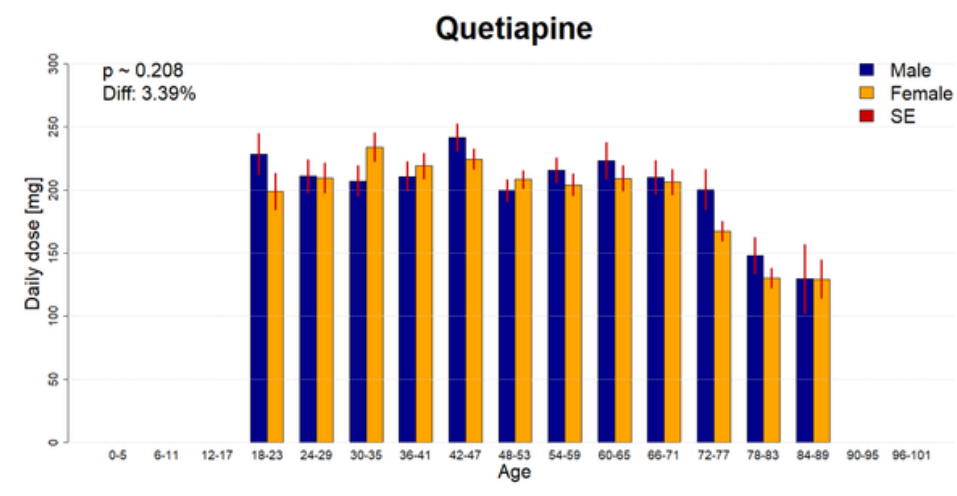

(b) Quetiapine

\section{Figure 7}

Daily dose by age and sex: Antipsychotics Daily prescribed doses $(\mathrm{mg})$ of antipsychotics by age: males in blue and female in orange. The standard error is depicted in red. The height of the bars corresponds to the mean daily dosage for inpatients within age groups of three consecutive years. The patients are between 18 and 89 years old. P-value: The p-value is computed from a (paired) Wilcoxon signed-rank test under the hypothesis that there are no differences between sexes. Diff: The percentage difference between sexes. Female patients are chosen as reference. From the plots can be seen, that the prescribed dosage differences between men and females are either small or statistically insignificant: $p<0.05$ for olanzapine, n.s. for quetiapine. 


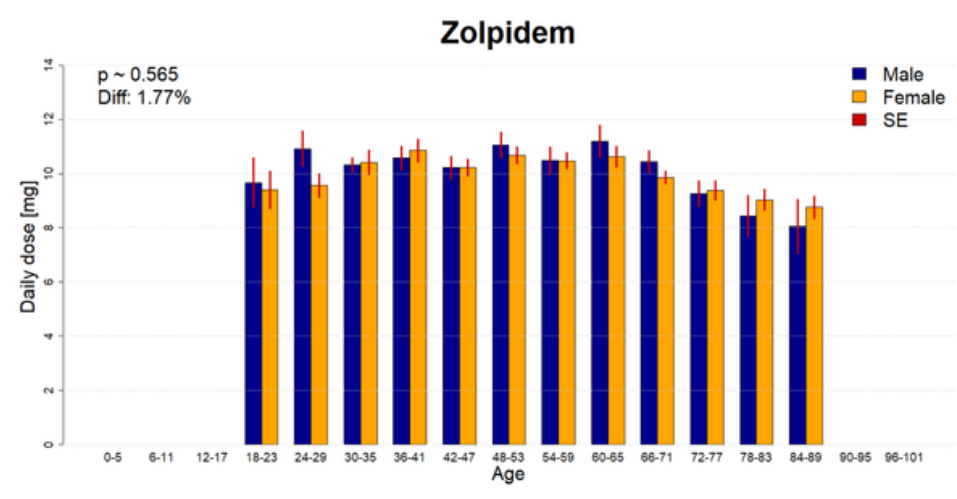

(a) Zolpidem

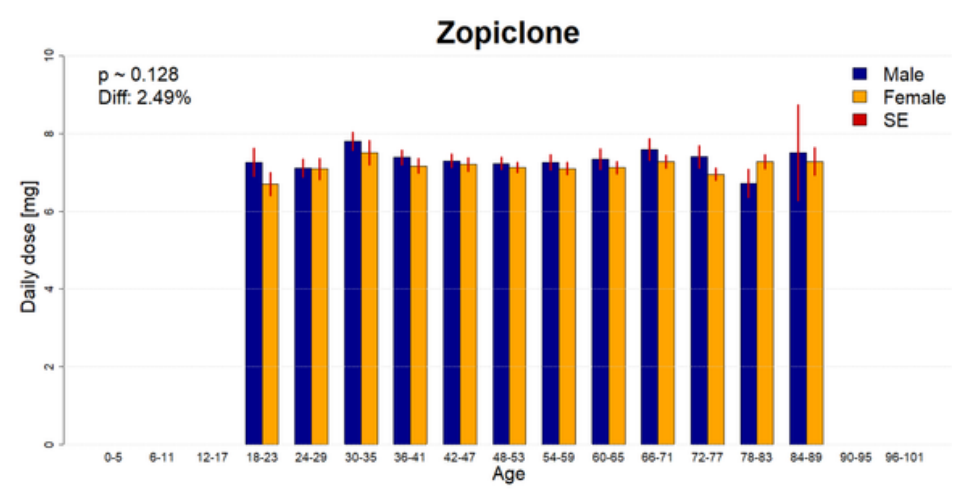

(b) Zopiclone

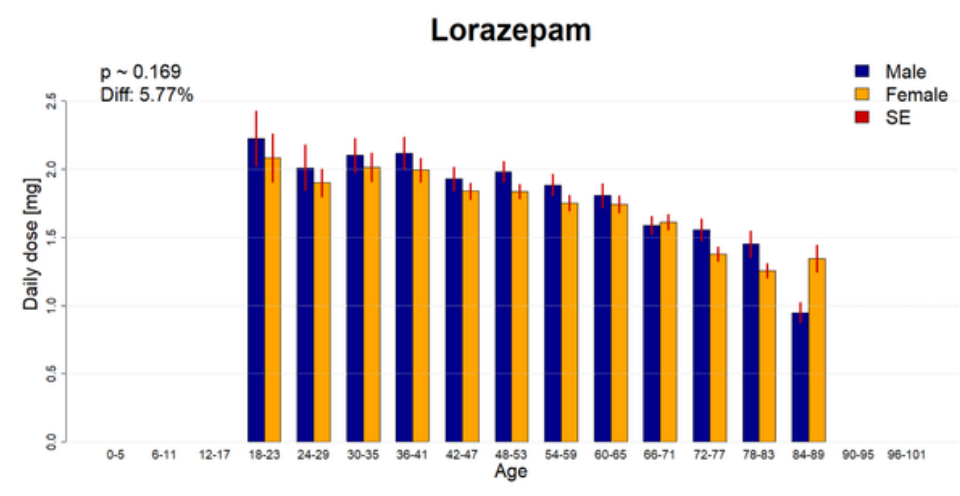

(c) Lorazepam

\section{Figure 8}

Daily dose by age and sex: Hypnotics and Tranquilizers Daily prescribed doses $(\mathrm{mg})$ of hypnotics and tranquilizers by age: males in blue and female in orange. The standard error is depicted in red. The height of the bars corresponds to the mean daily dosage for inpatients within age groups of three consecutive years. The patients are between 18 and 89 years old. P-value: The $p$-value is computed from a (paired) Wilcoxon signed-rank test under the hypothesis that there are no differences between sexes. Diff: The percentage difference between sexes. Female patients are chosen as reference. From the plots can be seen, that the prescribed dosage differences between men and females are small and statistically insignificant. 


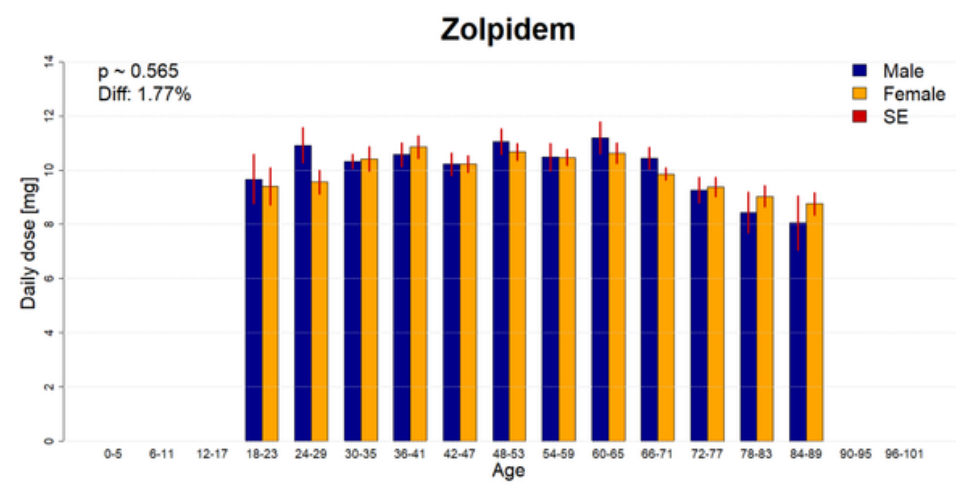

(a) Zolpidem

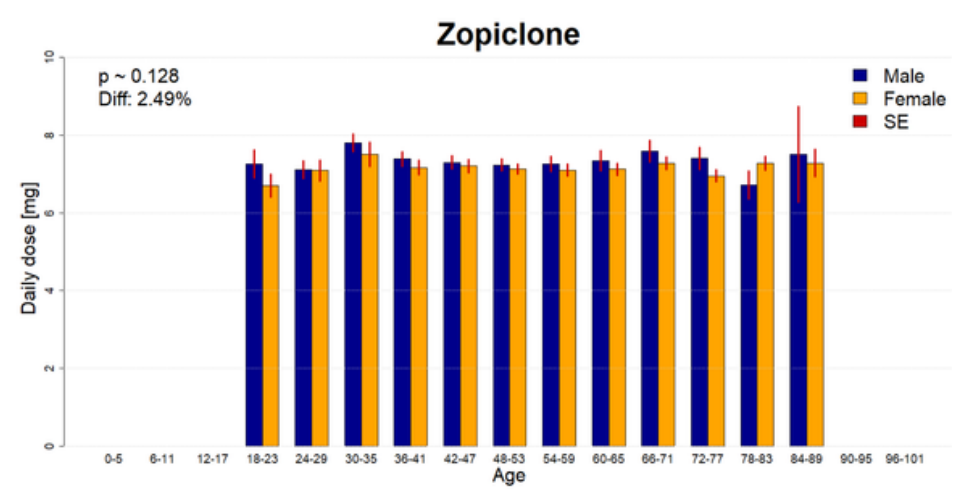

(b) Zopiclone

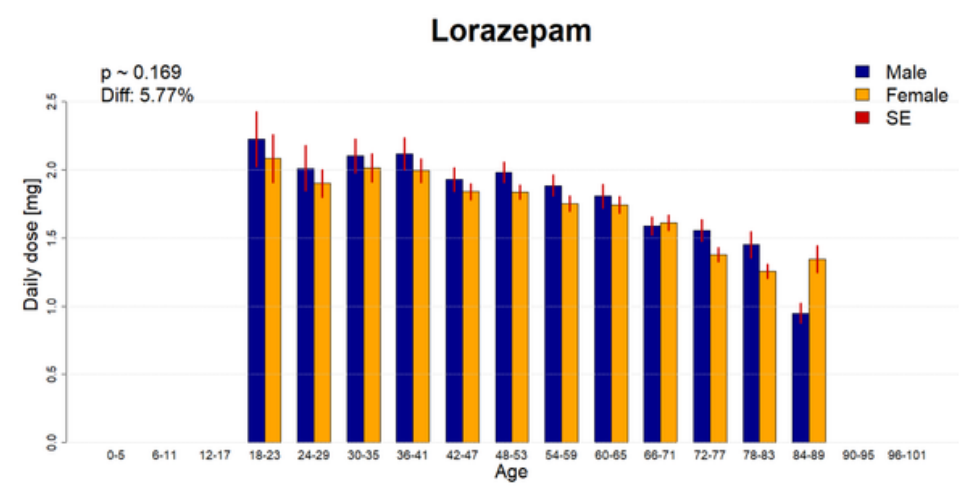

(c) Lorazepam

\section{Figure 8}

Daily dose by age and sex: Hypnotics and Tranquilizers Daily prescribed doses $(\mathrm{mg})$ of hypnotics and tranquilizers by age: males in blue and female in orange. The standard error is depicted in red. The height of the bars corresponds to the mean daily dosage for inpatients within age groups of three consecutive years. The patients are between 18 and 89 years old. P-value: The $p$-value is computed from a (paired) Wilcoxon signed-rank test under the hypothesis that there are no differences between sexes. Diff: The percentage difference between sexes. Female patients are chosen as reference. From the plots can be seen, that the prescribed dosage differences between men and females are small and statistically insignificant.

\section{Supplementary Files}

This is a list of supplementary files associated with this preprint. Click to download.

- 201127GREILAdditionalfiles.docx

- 201127GREILAdditionalfiles.docx 